\title{
LATE TIME MULTI-WAVELENGTH OBSERVATIONS OF SWIFT J1644+5734: A LUMINOUS OPTICAL/IR BUMP AND QUIESCENT X-RAY EMISSION
}

\author{
A. J. Levan ${ }^{1}$, N. R. Tanvir ${ }^{2}$, G. C. Brown ${ }^{1}$, B. D. Metzger ${ }^{3}$, K. L. Page ${ }^{2}$, S. B. Cenko ${ }^{4,5}$, P. T. O’Brien ${ }^{2}$, J. D. Lyman ${ }^{1}$, \\ K. Wiersema ${ }^{2}$, E. R. Stanway ${ }^{1}$, A. S. Fruchter ${ }^{6}$, D. A. Perley ${ }^{7,8}$, and J. S. Bloom ${ }^{9}$ \\ ${ }^{1}$ Department of Physics, University of Warwick, Coventry, CV4 7AL, UK; A.J.Levan@warwick.ac.uk \\ ${ }^{2}$ Department of Physics and Astronomy, University of Leicester, Leicester, LE1 7RH, UK \\ ${ }^{3}$ Columbia Astrophysics Laboratory, New York, NY 10027, USA \\ ${ }^{4}$ Astrophysics Science Division, NASA Goddard Space Flight Center, Mail Code 661, Greenbelt, MD 20771, USA \\ 5 Joint Space-Science Institute, University of Maryland, College Park, MD 20742, USA \\ ${ }^{6}$ Space Telescope Science Institute, 3700 San Martin Drive, Baltimore, MD 21218, USA \\ ${ }^{7}$ Department of Astronomy, California Institute of Technology, MC 249-17, 1200 East California Boulevard, Pasadena, CA 91125, USA \\ ${ }^{8}$ Dark Cosmology Centre, Niels Bohr Institute, DK-2100 Copenhagen, Denmark 0000-0002-4571-2306 \\ ${ }^{9}$ Astronomy Department, University of California, Berkeley, CA 94720-7450, USA \\ Received 2015 September 28; accepted 2015 December 23; published 2016 February 26
}

\begin{abstract}
We present late time multi-wavelength observations of Swift J1644+57, suggested to be a relativistic tidal disruption flare (TDF). Our observations extend to $>4$ years from discovery and show that 1.4 years after outburst the relativistic jet switched off on a timescale less than tens of days, corresponding to a power-law decay faster than $t^{-70}$. Beyond this point weak X-rays continue to be detected at an approximately constant luminosity of $L_{\mathrm{X}} \sim 5 \times 10^{42} \mathrm{erg} \mathrm{s}^{-1}$ and are marginally inconsistent with a continuing decay of $t^{-5 / 3}$, similar to that seen prior to the switch-off. Host photometry enables us to infer a black hole mass of $M_{\mathrm{BH}}=3 \times 10^{6} M_{\odot}$, consistent with the late time X-ray luminosity arising from sub-Eddington accretion onto the black hole in the form of either an unusually optically faint active galactic nucleus or a slowly varying phase of the transient. Optical/IR observations show a clear bump in the light curve at timescales of 30-50 days, with a peak magnitude (corrected for host galaxy extinction) of $M_{R} \sim-22$ to -23 . The luminosity of the bump is significantly higher than seen in other, nonrelativistic TDFs and does not match any re-brightening seen at X-ray or radio wavelengths. Its luminosity, light curve shape, and spectrum are broadly similar to those seen in superluminous supervnovae, although subject to large uncertainties in the correction of the significant host extinction. We discuss these observations in the context of both TDF and massive star origins for Swift J1644+5734 and other candidate relativistic tidal flares.
\end{abstract}

Key words: galaxies: active - galaxies: jets - supernovae: general

\section{INTRODUCTION}

Recent years have seen the discovery of new populations of extremely long duration $\gamma$-ray transients, visible for hours to days compared to seconds or minutes, for the well studied populations of gamma-ray bursts (GRBs; e.g., Burrows et al. 2011; Levan et al. 2011, 2014; Gendre et al. 2013). These events stretch plausible progenitor models for normal GRBs that arise from stellar core collapse and in particular, the longest events have been well explained by the tidal disruption of stars by supermassive black holes, accompanied by a moderately relativistic outflow, creating a $\gamma$-ray transient when viewed down the jet (Bloom et al. 2011b; Burrows et al. 2011; Zauderer et al. 2011).

Tidal disruption flares (TDFs) occur when a star strays sufficiently close to a massive black hole that the tidal force from the hole exceeds the star's self-gravity. At this point the star may be partly or completely disrupted depending on the pericenter separation and structure of the star itself. Roughly half of the disrupted material is expelled, while the remaining bound material is placed on eccentric orbits but eventually returns to form an accretion disk around the black hole, powering a luminous electromagnetic transient, with a blackbody spectrum expected to peak in the EUV or soft X-ray regime (e.g., Rees 1988). This process effectively creates a transient active nucleus, which, unlike most active galactic nuclei (AGNs), moves from a quiescent accretion phase through a super-Eddington one and back to quiescence on a timescale of years.

The detection of a TDF provides both a window on accretion physics and a signal of the presence of a supermassive black hole in an otherwise inactive galaxy. This is particularly valuable for low-mass galaxies where direct confirmation of massive black holes has proved challenging. While some massive black holes have been identified within dwarf galaxies (e.g., Reines et al. 2011, 2014) their interpretation remains uncertain: some lie apparently well off the bulge mass-black hole mass relation (e.g., Seth et al. 2014), and it is unclear if these rare examples are representative of other dwarfs (where no activity can be found) or result from unusual interactions such as the tidal stripping of more massive galaxies (Reines 2014; Seth et al. 2014). TDFs can occur, in principle, around all low-mass black holes, while they will be observationally invisible for the most massive systems $M_{\mathrm{BH}}>10^{8} M_{\odot}$ where the tidal radius for a main-sequence star lies within the Schwarzschild radius of the black hole. Thus they provide a particularly powerful probe of the low-mass end of the nuclear black hole population (e.g., Metzger \& Stone 2015), potentially extending down to the scale of intermediate mass black holes within globular clusters (e.g., Ramirez-Ruiz \& Rosswog 2009; MacLeod et al. 2015a, 2015b), and offer important constraints on models of galaxy formation and evolution.

However, TDFs themselves are challenging to locate and identify. They are much rarer than supernovae; they reside in 
regions of high surface brightness that are often omitted or difficult to recover in current transient surveys and the TDF population itself may exhibit significant diversity. For example, emission may arise from the disk, from stream collisions, from an outflow, or from an aligned relativistic jet. All of these events may appear observationally distinct, particularly due to viewing angle effects and hence difficult to distinguish from alternative types of transient. Thus, while there are several candidate TDFs reported in the literature (see, e.g., Komossa 2015 for a recent review), it remains unclear how many really represent tidal flares. Furthermore, the small samples and various mechanisms of discovery is such that it is not yet possible to utilize the observed population of candidate flares to infer the ubiquity and demographics of massive black holes within the nuclei of different types of galaxy.

A new chapter in this field began in 2011 March with the discovery of Swift J1644+57, a high-energy transient unlike any system seen before. It originated from the nucleus of a compact galaxy at $z=0.35$ (Levan et al. 2011), but its $\gamma$-ray emission persisted for days at the $10^{47} \mathrm{erg} \mathrm{s}^{-1}$ level (isotropic equivalent luminosity). It also exhibited an extremely longlived X-ray counterpart (Burrows et al. 2011; Levan et al. 2011), which remained at a luminosity brighter than $10^{45} \mathrm{erg} \mathrm{s}^{-1}$ for more than a year post-outburst. At first sight these properties do not obviously match the expectations for a TDF. First, the peak luminosity of Swift J1644+57 exceeds the Eddington limit for even a $10^{10} M_{\odot}$ black hole. It is highly unlikely that this galaxy hosts such a black hole since its apparent total stellar mass is less than this value (Levan et al. 2011; Yoon et al. 2015). Indeed, we would not expect to observe disruptions of main-sequence stars around such massive black holes. Hence the emission, if isotropic, must be super-Eddington by a factor of 100 or more. Second, TDFs are expected to be dominated by thermal (or near thermal) emission with temperature of a few $\times 10^{4} \mathrm{~K}$, while the emission from Swift J1644+57 was apparently dominated by a much harder, power-law component enabling its detection by the $\gamma$-ray detectors on board Swift (Bloom et al. 2011b; Burrows et al. 2011).

Soon after its discovery, it was proposed that these properties could be naturally explained if Swift $\mathrm{J} 1644+57$ was due to relativistically jetted emission from a tidal disruption event (Bloom et al. 2011a, 2011b). In fact, a scenario in which some small fraction of the material from a TDF was expelled at relativistic velocities had already been considered, but primarily from the point of view of possible late time radio emission from known TDFs, which may become visible at the point the blast wave is approximately spherical (Giannios \& Metzger 2011; van Velzen et al. 2011). These authors did not consider what may happen when one views directly down the relativistic jet since this chance alignment is unlikely. However, this low space density is compensated (at least to some degree, depending on the beaming angle) by the luminosity, providing a much larger horizon over which these events may be seen. Given this, Bloom et al. (2011b) suggested that Swift J1644 +57 was in fact such an event, effectively a micro-blazar. Subsequent precise astrometry (Levan et al. 2011), the general shape of the X-ray light curve, and the direct measurement of relativistic expansion via radio observations offer substantial support for this scenario (Bloom et al. 2011b; Zauderer et al. 2011). Remarkably, despite seeing none of these events in the first six years of this mission, a second possible example,
Swift J2058+0516, was uncovered in 2011 May (Cenko et al. 2012b), and a third, Swift J1112-8238 (Brown et al. 2015), although only recently recognized, was detected in 2011 June. It is clear that these events are set apart from previously identified TDFs, perhaps because of the impact of viewing angle (Bloom et al. 2011b) although also perhaps because of unique features of the disrupting system such as a deeply plunging orbit largely destroying the star (Cannizzo et al. 2011) or binarity (Mandel \& Levin 2015).

However, alternative hypotheses have also been considered for these systems. Specifically, it has been suggested that they could arise from the core collapse of massive stars in systems not unlike those which create long duration gamma-ray bursts (Quataert \& Kasen 2012; Woosley \& Heger 2012). The basic model for creating such events is that material in the outer layers of a rotating massive star has too much angular momentum to collapse directly onto the nascent compact object and instead forms an equatorial disk, which feeds the newly formed black hole for a long period of time. These events differ from traditional GRBs because it is not the material immediately outside the collapsing core forming a relatively short-lived disk but material initially at much larger distances, creating more massive, long-lived accretion events. These models were not fully developed until after the initial discovery of Swift J1644+57 and are not obviously favored given the nuclear location of the transient seen in both Swift J1644+57 (Levan et al. 2011) and Swift J2058+0516 (Pasham et al. 2015) and possibly (though not conclusively) in Swift J1112-8238 (Brown et al. 2015). However, to date, no conclusive evidence against (or in favor) of them has been found. Interestingly, similar models have been postulated to explain the origin of the ultra-long GRBs (with durations around $10^{4} \mathrm{~s}$ (Levan et al. 2014)) where giant star models have had some success (Gendre et al. 2013; Nakauchi et al. 2013; Stratta et al. 2013; Levan et al. 2014). Indeed the recent identification of a luminous supernova in the afterglow of the ultra-long GRB 111209A (duration $\sim 2 \times 10^{4}$ s) does apparently demonstrate that core collapse, GRB-like explosions can occur with durations at least an order of magnitude longer than seen in most GRBs (Greiner et al. 2015).

Here we present late time observations of the best studied event, Swift J1644+57, at wavelengths from the X-ray to the mid-IR, spanning from 30 days to 4 years after the detection of the initial outburst. We use these to characterize the light curves and host galaxy. Three striking features are seen: (i) a rapid drop in the X-ray luminosity 500 days post-outburst, as also noted by Sbarufatti et al. (2012), Levan \& Tanvir (2012), Zauderer et al. (2013), and Mangano et al. (2015); (ii) an apparently quiescent underlying X-ray source of luminosity $L_{\mathrm{X}} \sim 5 \times 10^{42} \mathrm{erg} \mathrm{s}^{-1}$, consistent with a low-luminosity AGN; and (iii) a pronounced bump in the optical/IR light curves, peaking 30-40 days after the initial outburst, with an absolute magnitude of $M_{V} \sim-22$. We discuss these properties in light of the expectations of various models for the creation of these extreme high-energy transients.

\section{OBSERVATIONS}

Swift J1644+57 was discovered by the Swift-BAT on 2011 March 28 (Cummings et al. 2011). Initially classified as a GRB (GRB 110328A) the detection of additional bright flares in the following $48 \mathrm{hr}$ period (Suzuki et al. 2011) and the subsequent discovery of emission in a 4 day window prior to the initial 
Table 1

Ground-based Photometric Observations of Swift J1644+57

\begin{tabular}{|c|c|c|c|c|c|c|}
\hline Date-obs & MJD-obs & $\begin{array}{c}\Delta T \\
\text { (days) }\end{array}$ & Telescope & Band & $\begin{array}{c}\text { Exptime } \\
\text { (s) }\end{array}$ & $\begin{array}{c}\text { Mag } \\
\text { (host+OT) }\end{array}$ \\
\hline 2011 Aug 02 & 55775.26 & 126.73 & UKIRT/WFCAM & $\mathrm{K}$ & 1800 & $20.72 \pm 0.07$ \\
\hline 2011 Aug 02 & 55775.26 & 126.76 & UKIRT/WFCAM & $\mathrm{J}$ & 2160 & $21.52 \pm 0.03$ \\
\hline 2011 Oct 07 & 55841.24 & 192.70 & UKIRT/WFCAM & $\mathrm{K}$ & 2160 & $20.53 \pm 0.17$ \\
\hline 2011 Oct 09 & 55843.20 & 194.66 & UKIRT/WFCAM & $\mathrm{K}$ & 2160 & $20.94 \pm 0.08$ \\
\hline 2011 Oct 09 & 55843.24 & 194.70 & UKIRT/WFCAM & $\mathrm{J}$ & 2160 & $21.84 \pm 0.23$ \\
\hline 2011 Oct 10 & 55844.20 & 195.66 & UKIRT/WFCAM & $\mathrm{H}$ & 2160 & $21.57 \pm 0.20$ \\
\hline 2012 Mar 13 & 55999.64 & 351.10 & Gemini/GMOS & $\mathrm{r}$ & 900 & $22.49 \pm 0.02$ \\
\hline 2012 Mar 13 & 55999.66 & 351.12 & Gemini/GMOS & $\mathrm{z}$ & 900 & $21.94 \pm 0.02$ \\
\hline 2012 May 02 & 56049.45 & 400.91 & Gemini/NIRI & $\mathrm{K}$ & 1560 & $21.42 \pm 0.04$ \\
\hline 2012 May 02 & 56049.47 & 400.93 & Gemini/NIRI & $\mathrm{H}$ & 1560 & $21.83 \pm 0.09$ \\
\hline 2012 May 02 & 56049.49 & 400.95 & Gemini/NIRI & $\mathrm{J}$ & 1560 & $21.99 \pm 0.11$ \\
\hline 2012 Jun 30 & 56108.39 & 459.85 & Gemini/NIRI & $\mathrm{J}$ & 2280 & $21.90 \pm 0.06$ \\
\hline 2012 Jun 30 & 56108.43 & 459.89 & Gemini/NIRI & $\mathrm{H}$ & 1560 & $21.63 \pm 0.04$ \\
\hline 2012 Jun 30 & 56108.46 & 459.92 & Gemini/NIRI & $\mathrm{K}$ & 2040 & $21.30 \pm 0.05$ \\
\hline 2012 Oct 11 & 56211.20 & 592.66 & UKIRT/WFCAM & $\mathrm{K}$ & 1800 & $21.22 \pm 0.12$ \\
\hline
\end{tabular}

Note. Magnitudes are not host subtracted. This table supplements the photometry given in Levan et al. (2011).

detection (Krimm \& Barthelmy 2011) marked it as having exceptionally long $\gamma$-ray emission, persisting for several days (see also Levan et al. 2014). Indeed, a possible detection at $>3 \sigma$ significance was present in a single day integration $>1$ month before the main trigger (Krimm \& Barthelmy 2011). While possibly a chance noise fluctuation, it is interestingly close to the time of the first trigger so earlier activity cannot be discounted. Although initially suggested to be a Galactic X-ray transient (Kennea et al. 2011), a redshift of a persistent optical source underlying the X-ray location revealed a redshift of $z=0.354$ (Levan et al. 2011) and subsequent monitoring located X-ray, infrared, and radio emission consistent with the nucleus of this galaxy. The early observations have been described in detail (Bloom et al. 2011b; Burrows et al. 2011; Levan et al. 2011; Zauderer et al. 2011) and the source has continued to be monitored by the Swift-XRT. The late time radio afterglow has also received significant monitoring (Berger et al. 2012; Zauderer et al. 2013). Below we report the results of ongoing late time optical/IR and X-ray monitoring from both the ground and space.

\subsection{Further Infrared and Optical Imaging}

We have continued to monitor Swift J1644+57 in the IR from the United Kingdom Infrared Telescope (UKIRT) and Gemini-North. A log of our new photometric observations is shown in Table 1. The UKIRT images were obtained with the Wide Field Camera (WFCAM) and reduced through the standard CASU pipeline. The data were retrieved in calibrated form from the WFCAM science archive. ${ }^{10}$ The Gemini-North images were reduced using the standard Gemini IRAF package. Photometric calibration was performed relative to several 2MASS stars, with the zeropoint tied to the star at R.A. $=16: 44: 50.96, \quad$ decl. $=+57: 35: 31.6 \quad(J=13.121$, $H=12.798, K=12.727$ ) as in Levan et al. (2011) such that the photometric observations should be directly comparable between earlier work and this one.

We also include in our analysis other published IR photometry from Burrows et al. (2011). Observations taken

\footnotetext{
${ }^{10}$ http://wsa.roe.ac.uk/
}

at similar times provide reasonable agreement with our measured photometry within $\sim 0.1-0.2 \mathrm{mag}$ and hence should be on a comparable scale. There is no apparent systematic offset that could be applied to reduce this scatter significantly, and so it is likely that the differences in measurements reflect a combination of measurement error (often significant at later times) and true variation within the source (often significant at earlier times).

\subsection{HST and Spitzer Observations}

We have also obtained further observations of Swift J1644 +57 with the Hubble Space Telescope (HST). These observations were obtained in the F606W and F160W bands using the WFC3 camera with both UVIS and IR channels, matching the earlier data presented in Levan et al. (2011). The images were retrieved from the archive after standard post-processing. The UVIS observations were corrected for pixel-dependent CTE utilizing the method of Anderson (2014). The images were then drizzled (Fruchter \& Hook 2002) onto a common frame, utilizing a pixel scale of $0.025 \operatorname{arcsec}_{\text {pixel }}^{-1}$ for $\mathrm{F} 606 \mathrm{~W}$ and 0.07 arcsec pixel $^{-1}$ for F160W. The first and last epochs, as well as a subtraction, are shown in Figure 1. To obtain magnitudes of the counterpart only the final epoch of HST observations was subtracted from the earlier data and the resulting residual measured. The photometry is shown in Table 2, where both transient fluxes and combined host plus transient magnitudes are listed. To avoid including additional sky noise, which may impair the estimation of transient contributions, the combined magnitudes were measured in an aperture of radius 15 pixels for F160W (1".05) and corrected assuming a pointlike aperture correction. In practice this underestimates the true host galaxy magnitude, and so the host galaxy magnitude itself is calculated based on the Sérsic profile fit to the host galaxy, yielding a magnitude approximately 0.2 mag brighter. The resulting magnitudes for the host galaxy are comparable to those obtained by Yoon et al. (2015) from an independent analysis of our data. The relatively bright point sources in subtractions were measured in small apertures $(2 \times$ FWHM $)$, and aperture corrected, while due to possible galaxy residuals we measured the F606W subtractions in 

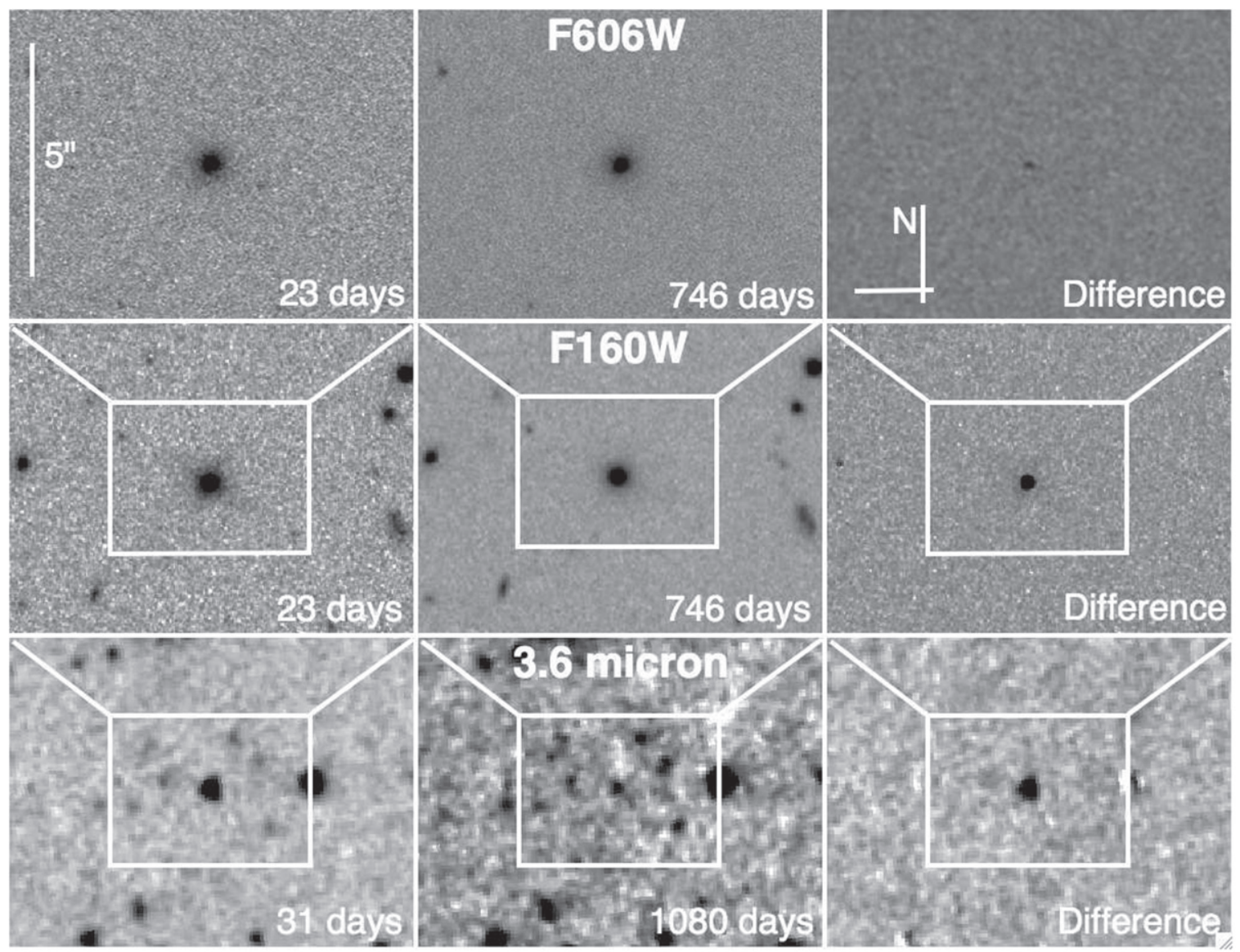

Figure 1. Early to late time space-based observations of Swift J1644+57 with HST and Spitzer. At early times the NIR and mid-IR are dominated by afterglow emission, while in the optical the host dominates at all epochs, although a weak transient can be seen in our F606W observations.

Table 2

Late-time Space-based Optical/IR/MIR Observations of Swift J1644+57

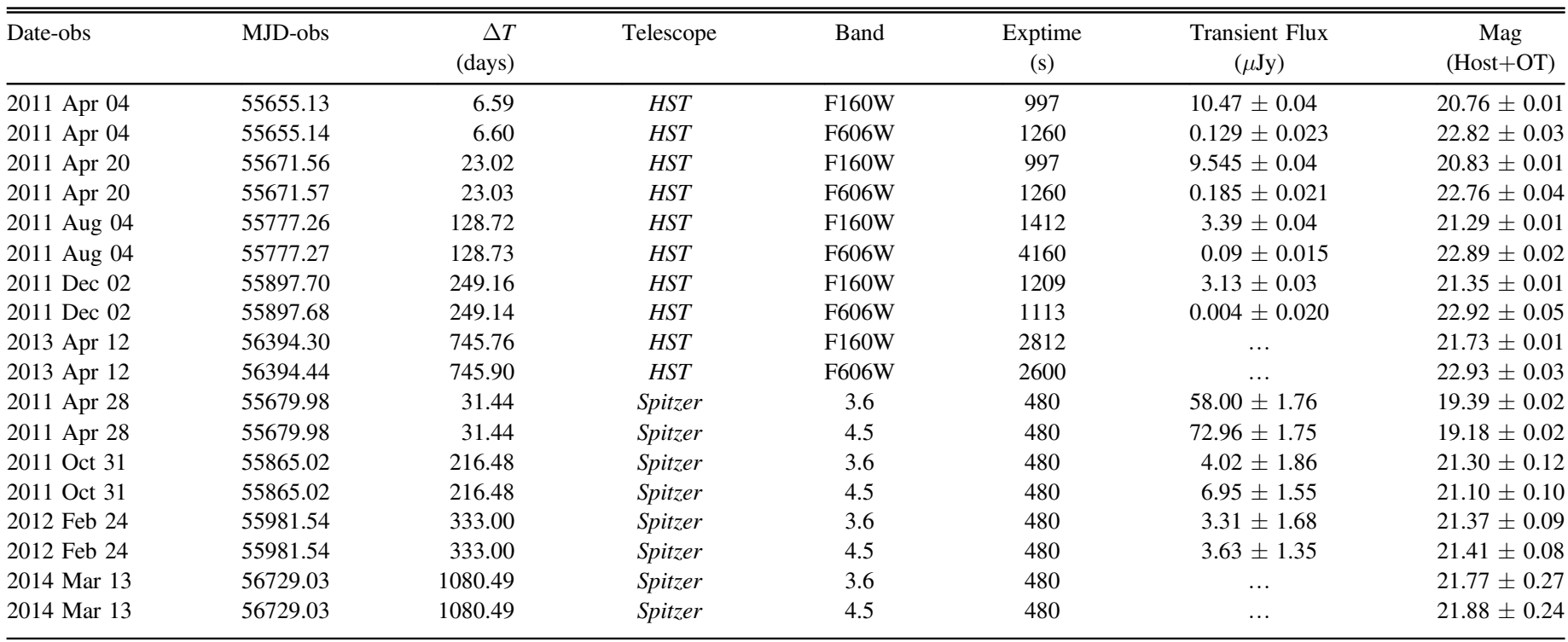

Note. Log of late time observations of Swift J1644+57 obtained with the Hubble Space Telescope and the Spitzer Space Telescope. Photometry is listed with and without host subtraction.

apertures of $0 . " 4$. We note that as expected the choice of aperture size has little impact on our final photometry.

A clear residual is seen in both bands. In fact, this is the first detection of transient optical emission in the r-band, previous detections having only been possible in the $\mathrm{z}$ band and longwards (Levan et al. 2011), likely due to the strong extinction within the host galaxy. Interestingly, the optical light appears to rise between the first two epochs (6.6 and 23 days 
Table 3

Late Time X-Ray Observations of Swift J1644+57

\begin{tabular}{|c|c|c|c|c|c|c|}
\hline Date-obs & MJD-obs & $\begin{array}{c}\Delta T \\
\text { (days) }\end{array}$ & Telescope & ks & $\begin{array}{c}\text { Count Rate } \\
(0.3-10 \mathrm{keV})\end{array}$ & $\begin{array}{c}\text { Flux } \\
\left(\mathrm{erg} \mathrm{s}^{-1} \mathrm{~cm}^{-2}\right)\end{array}$ \\
\hline 2012 Sep 27 & 56197.81 & 549.27 & XMM & 22.7 & $(1.9 \pm 0.3) \times 10^{-3}$ & $9.93 \times 10^{-15}$ \\
\hline 2012 Oct 05 & 56205.80 & 557.26 & XMM & 28.7 & $(1.2 \pm 0.2) \times 10^{-3}$ & $6.27 \times 10^{-15}$ \\
\hline 2012 Nov 26 & 56257.44 & 608.90 & Chandra & 24.7 & $(3.0 \pm 1.1) \times 10^{-4}$ & $4.18 \times 10^{-15}$ \\
\hline 2013 Jul 17 & 56490.70 & 842.16 & XMM & 44.1 & $(8.1 \pm 1.5) \times 10^{-4}$ & $4.21 \times 10^{-15}$ \\
\hline 2015 Feb 17 & 57070.20 & 1421.66 & Chandra & 27.8 & $(4.6 \pm 1.3) \times 10^{-4}$ & $6.40 \times 10^{-15}$ \\
\hline 2015 Apr 06 & 57118.85 & 1470.31 & Chandra & 18.7 & $(2.7 \pm 1.3) \times 10^{-4}$ & $3.76 \times 10^{-15}$ \\
\hline 2012 Sep 02 & 56172.86 & $524.32_{-17}^{+34}$ & XRT & 110.7 & $(1.90 \pm 0.69) \times 10^{-4}$ & $9.19 \times 10^{-15}$ \\
\hline 2013 Mar 15 & 56366.85 & $718.31_{-160}^{+251}$ & XRT & 146.4 & $(1.61 \pm 0.67) \times 10^{-4}$ & $7.79 \times 10^{-15}$ \\
\hline
\end{tabular}

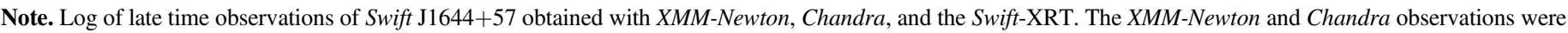

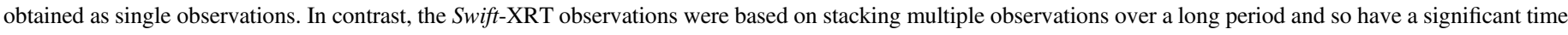
error bar. The dates given for these observations correspond to the $\Delta T$ given, as determined by the methods described in Evans et al. (2007, 2010).

post-outburst) during which time the IR appears to show a decline. This is puzzling if both the optical and IR are arising from the same component and is discussed further below.

We can determine the location of the transient within the host galaxy by comparing the centroid in the subtracted frames with the center of the host galaxy in late epoch images, utilizing compact sources in the field for astrometric purposes. This is best done in the IR since the signal to noise for the transient is much higher, does not risk any systematic shift due to poorer subtraction of the host galaxy light, and minimizes the risk of mis-identifying the centroid due to differential extinction within the host galaxy. We compared our first and last epoch, using eight sources in common between the two frames for alignment. As the first and last images were taken at the same orientation we can utilize a direct shift between the two rather than more complex fits (which may underestimate the errors for the small number of sources considered). This yields an offset of $(0.010 \pm 0.012)^{\prime \prime}$, equivalent to a spatial offset of $<60 \mathrm{pc}$ from the centroid of the galaxy. Although it has limitations this approach can also be used in the F606W observations, which yields an offset of $(0.033 \pm 0.010)^{\prime \prime}$. This is formally inconsistent with the nucleus at the $3 \sigma$ level, but may be due to a combination of the effects described above. However, this technique is based on utilizing compact sources (predominantly stars) in the field of view and so proper motion can be a significant factor. A new technique, employing cross correlation with galaxies, can improve this and will be presented separately (R. Hounsell et al. 2015, in preparation).

We also observed Swift J1644+57 with the Spitzer Space Telescope at four epochs. The first three roughly span a year after the outburst, with a final epoch obtained in 2014 March for host subtraction. Observations were obtained in both the 3.6 and $4.5 \mu \mathrm{m}$ bands. Photometry was performed directly on the PBCD mosaics and on aligned and subtracted images to isolate the transient flux utilizing a 4 pixel ( 2 native pixel) aperture and correcting for excluded light. The IRAC observations suggest a bright mid-IR outburst, consistent with a highly extinguished source, which fades by by a factor of 10 over the course of the first year. A log of observations and resulting photometry is shown in Table 2.

\subsection{Host Galaxy Spectroscopy}

In addition to the early spectroscopy reported in Levan et al. (2011) we obtained further optical spectroscopy with GeminiN/GMOS on 2011 July 23 and 2012 March 23 to April 4.
Observations were obtained in the R400 filter spanning a wavelength range from $\sim 5900$ to $10000 \AA$ and utilizing the nod-and-shuffle mode to improve sky subtraction. The data were reduced via the Gemini GMOS pipeline appropriate for simple longslit (for our earlier observations) or nod and shuffle (for later data). The previously reported emission lines of $\mathrm{H} \alpha$, $\mathrm{H} \beta$, [O III], and [O II] (Levan et al. 2011) remain visible and no clear evolution is seen. In particular, the lines remain narrow with no sign of the development of broad lines around $\mathrm{H} \alpha$, where some recently identified TDF candidates have shown transient broad features (Gezari et al. 2012; Arcavi et al. 2014). This is unsurprising given the low level of broadband optical variability in the source and may be indicative of a lack of broad features, or could suggest that the lines seen are from relatively unobscured star formation within the host galaxy, while any broadline region remains highly obscured.

\subsection{Late Time X-Ray Observations With XMM-Newton and Chandra}

We obtained several epochs of late time observations of Swift J1644+57 with both XMM-Newton and Chandra. A log of these observations with exposure times is shown in Table 3. All XMM-Newton observations utilized the thin filter for both PN and MOS observations. Chandra observations used ACIS$\mathrm{S}$ in very faint mode with the source placed at the default aim point on the S3 chip.

For our Chandra observations we extracted images from the cleaned event files in the $0.3-10 \mathrm{keV}$ energy band. We then determined count rates in an aperture of $2^{\prime \prime}$ radius. Although faint, the source is detected in each individual image with between 7 and 17 source counts and within our aperture the background is negligible ( $<1$ count expected). Given the small number of counts it is not possible to determine detailed spectral parameters for our data, although as noted by Berger et al. (2012) the X-ray photons arise across the energy range and are not dominated by soft photons as would be expected for a thermal blackbody typically thought to underly TDFs.

The XMM-Newton data were reduced with SAS 14.0.0 using EPCHAIN and EMCHAIN to extract the event lists. All the XMMNewton observations utilized the thin filter for both pn and MOS observations; single- and double-pixel events (patterns 0-4) for pn and all events up to quadruple pixels (patterns 0-12) for MOS were selected. The event lists were screened for times of high, flaring background and an energy range of $0.3-10 \mathrm{keV}$ was then considered. Source count rates were 

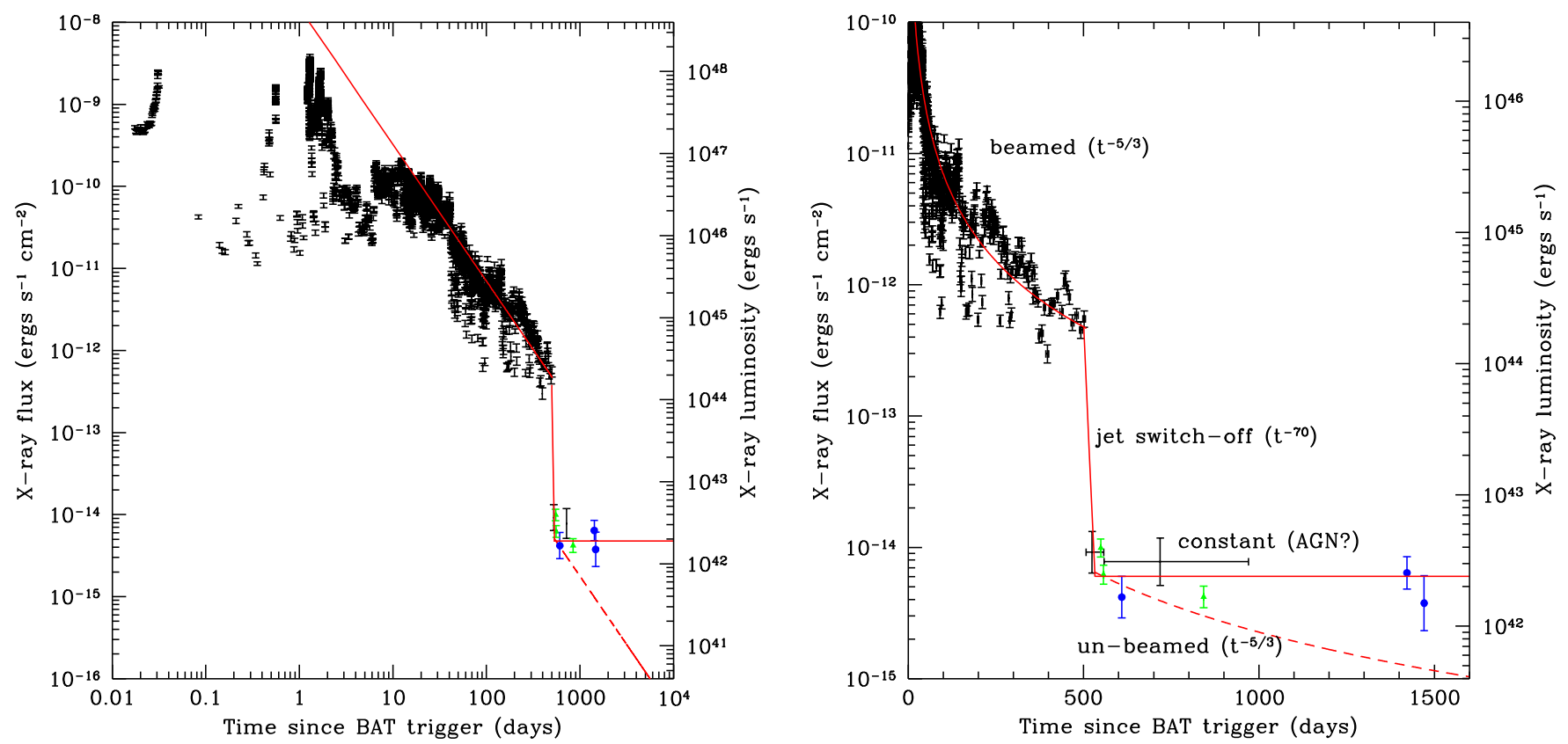

Figure 2. X-ray light curve of Swift J1644+57 obtained with the Swift-XRT (black), XMM-Newton (green), and Chandra (blue). The data plotted in each fit are identical but are plotted on a logarithmic (left) and linear (right) scale to emphasize both the overall shape and behavior after the rapid decay. The solid red line shows a $t^{-5 / 3}$ decay plotted through the X-ray observations. This is not a fit to the data, but an indicative reference model. A sharp break of $t^{-70}$ is shown at 500 days, followed by a constant level. For comparison, a continued decay of $t^{-5 / 3}$ after the end of the steep decline is shown as the dashed line.

extracted using a $10^{\prime \prime}$ radius circle centered on the source position and corrected for point-spread function (PSF) losses caused by the small region size. The background was estimated from a nearby, larger, source-free region. The numbers given in Table 3 for the XMM-Newton observations are from the pn data sets in each case.

We convert the measured X-ray count rates in the $0.3-10 \mathrm{keV}$ bands into fluxes assuming a simple model determined from the fit to the late time X-ray spectra measured by the Swift-XRT, namely, an absorbed power law of index $\Gamma=1.99$ and contributions from Galactic and host galaxy absorption $\quad\left(N_{\mathrm{H}, \mathrm{gal}}=1.75 \times 10^{20}, \quad N_{\mathrm{H}, \text { host }}=2.07 \times 10^{22}\right.$; (Willingale et al. 2013)). We note this does differ in detail from the fit found by more detailed spectral fitting when the source was brighter, which required an additional thermal component providing a few percent of the soft flux. However, the errors associated with the choice of spectrum are small compared to the photon counting errors for the source at this brightness. It is possible to fit the XMM-Newton PN observations directly since the combined observations contain 130 counts (of which approximately half are from the source). Doing so with the absorption fixed to the values determined by the XRT yields a power-law index of $\Gamma=1.85_{-0.73}^{0.51}$ (at $90 \%$ confidence), consistent with the earlier observations and implying no strong hard to soft evolution.

\section{DISCUSSION}

\subsection{Late Time X-Ray Light Curve}

The updated X-ray light curve of Swift J1644+57 is shown in Figure 2 on both logarithmic and linear time axes. Our late time observations have been supplemented by the ongoing observations with the Swift-XRT, taken from the Swift UK data

\footnotetext{
${ }^{11}$ The data presented are based on the calibrated XRT light curve available from the UKSSDC as of 2015 November 26.
}

center $^{11}$, processed via the techniques described in Evans et al. (2007, 2010). As previously noted (Bloom et al. 2011b; Burrows et al. 2011; Levan et al. 2011) the early light curve is dominated by pronounced flaring and variability, which then settles into a steady decay, punctuated by notable dips, which have been suggested to show some signs of periodicity (Saxton et al. 2012). The ongoing variability means that attempts to fit any simple decay model to the data inevitably lead to poor quality fits, although the data from $\sim 100-500$ days, if fit with a single power-law do favor a slope of $-5 / 3$ (Levan 2015). More complex fits could be attempted to investigate the presence or absence of additional breaks in the light curve, but these require some attempt to remove dipping activity, and so are necessarily limited in statistical power.

The final good detection reported by the Swift-XRT is at around 500 days, with a flux of $(5.5 \pm 0.8) \times 10^{-13}$ $\mathrm{erg} \mathrm{s}^{-1} \mathrm{~cm}^{-2}$, based on the stacking of images obtained $\sim 4$ days either side of this midpoint. After this, the X-ray flux decreased markedly. By the time of our XMM-Newton observations the source had declined by a factor of at least 50 in flux. In a factor of $\Delta T / T=0.08$ in time a fall of a factor of 50 corresponds to a decay index of around $t^{-70}$. In practice, the decay was too fast to be resolved since beyond the steep drop-off, XRT observations cannot recover the flux in short exposure times and there was a significant delay before the $X M M-N e w t o n$ and Chandra observations were scheduled. Hence we conclude that the power-law decay rate was faster than $t^{-70}$. Assuming we are observing X-ray activity from the base of the jet this suggests that activity suddenly shut off, due to either a switch of accretion mode or the cessation of accretion. Given the size of emitting regions at the head of the jet at this late time it is difficult to envision a scenario in which this shut off was not due to the cessation of activity close to the base of the jet since otherwise it would have smeared out over a much longer time period. 
It is interesting to note that such rapid cessation of X-ray activity was explicitly predicted in the massive star models of Quataert \& Kasen (2012) since this represents the point at which all of the star has accreted onto the central compact object. Such predictions were not made for jetted TDF-like events prior to the detection of the rapid drop in Swift J1644 +57 , although can potentially be explained via magnetic processes within the disk (Tchekhovskoy et al. 2014). In particular, once the black hole accretion rate becomes subEddington and radiatively efficient (geometrically thin), it enters a thermally dominant accretion state, which is empirically not observed to produce powerful jets in Galactic X-ray binaries (e.g., Russell et al. 2011).

After this rapid decay, X-rays of luminosity $L_{\mathrm{X}} \sim 5 \times 10^{42} \mathrm{erg} \mathrm{s}^{-1}$ continue to be detected until at least 2015 April (day 1500). These X-rays appear to be approximately constant in luminosity with little sign of a decay. A fit to the available Swift-XRT, XMM-Newton, and Chandra observations with a constant source is not especially good $\left(\chi^{2} /\right.$ dof $=13.7 / 7)$. The fit is not improved by allowing for a powerlaw model, which gives a best fit decay of $\alpha=0.5_{-0.2}^{+0.7}\left(\chi^{2} /\right.$ dof $=10.22 / 6$ ), with an F-test probability of chance improvement of $20 \%$. However, these data are dominated by observations immediately after the break and may contain additional systematic errors from comparison between three different instruments. If instead we compare the Chandra count rates then a constant source provides a very good description $\left(\chi^{2} / \mathrm{dof}=1.27 / 2\right)$, and the power-law slope of $\alpha=-0.2_{-0.4}^{+0.8}$ rules out a continuing decay around $t^{-5 / 3}$ at $>2.3 \sigma$ (and $t^{-4 / 3}$ at $1.9 \sigma$ ). This is at first sight surprising since it is reasonable to assume that after the cessation of jet activity we begin to observe forward shock emission at all wavelengths (Zauderer et al. 2013). The absence of continued decay of this emission would then suggest that these X-rays either do not originate from the forward shock or that it is somehow continuing to be energized despite the cessation of jet activity. It is hence interesting to compare this late time behavior to the general expectations of differing progenitor models.

In a TDF scenario, once the jet turns off, thermal X-ray emission from the inner accretion disk could be observed (which was originally considered the hallmark signature of TDFs; e.g., Rees 1988). For stellar tidal disruption by a black hole of mass $\sim 10^{6} M_{\odot}$, the fall-back time of the most tightly bound tidal debris (i.e., the time at which we expect accretion to start and an observable electromagnetic transient to be produced) is $t_{\mathrm{fb}} \sim 1$ month, similar to the duration of peak hard X-ray activity in Swift J1644+57 and J2058+05. The accretion luminosity is then some fraction, $\eta$, of the rest mass of the disrupted star $\left(M_{\star}\right)$ accreted. Since this mass returns as $\left.t / t_{\mathrm{fb}}\right)^{-5 / 3}$ (Rees 1988; Phinney 1989), this luminosity can be expressed as

$$
\begin{aligned}
L_{\mathrm{X}} \approx & \eta \dot{M} c^{2} \approx \eta \frac{M_{\star} c^{2}}{3 t_{\mathrm{fb}}}\left(\frac{t}{t_{\mathrm{fb}}}\right)^{-5 / 3} \\
\approx & 3 \times 10^{43} \mathrm{erg} \mathrm{s}^{-1}\left(\frac{\eta}{0.1}\right)\left(\frac{M_{\star}}{0.5 M_{\odot}}\right) \\
& \times\left(\frac{t_{\mathrm{fb}}}{\text { month }}\right)^{2 / 3}\left(\frac{t}{1000 \text { day }}\right)^{-5 / 3} .
\end{aligned}
$$

To order of magnitude, the predicted luminosity at $500-1000$ days is similar to that observed in $\mathrm{J} 1644+57$ after the steep drop (once a bolometric correction is included). However, the predicted $\propto t^{-5 / 3}$ decay is steeper than the observed light curve between 500 and 1000 days. A dimmer and flatter light curve than predicted by Equation (1) could be explained if the black hole accretion rate after the jet shut off no longer tracks the mass fall-back rate due to the viscous spreading of the disk (Cannizzo et al. 1990; Shen \& Matzner 2014). Such a transition from rapid to slow processing by the disk is naturally instigated by the sudden and large increase in the viscous timescale $\propto H^{-2}$ once the disk scale height $H$ shrinks following the subEddington state transition (Shen \& Matzner 2014). However, the apparently relatively hard X-ray spectrum after the rapid decay is not in keeping with the very soft thermal spectrum expected in TDFs, and so it seems less likely that this is the observed origin of the late time X-ray emission.

In the case where all the material from a collapsing star has been accreted (Quataert \& Kasen 2012) it seems unlikely that an essentially quiescent source would persist. One possibility is that some level of ongoing accretion may occur from the dense region in which the SN occurred, although the luminosity is orders of magnitude larger than possible from either Bondi-Hoyle accretion in a giant molecular cloud or from accretion from a companion star. Indeed, the luminosity of $\sim 5 \times 10^{42} \mathrm{erg} \mathrm{s}^{-1}$ remains $\sim 3$ orders of magnitude larger than possible from a stellar mass black hole, and would require both a continued high accretion rate and a significant degree of beaming unless the supernova had been from an extremely massive star that had created an exceptionally massive black hole (e.g., Portegies Zwart et al. 2004).

Finally, it is possible that the late time X-rays represent ongoing AGN activity, separate to the transient outburst. The $\mathrm{X}$-ray luminosity itself would be fairly typical for a lowluminosity AGN, however, the host galaxy would be unusual in this case since the majority of AGNs are hosted in rather more luminous galaxies. This is illustrated in Figure 3 which, following Levan et al. (2011), shows the comparative luminosity evolution of Swift J1644+57 in the X-ray luminosity against the optical/IR absolute magnitude plane. The track of the counterpart of Swift J1644+57 is shown at several characteristic times and shows that it evolves from extreme X-ray luminosity through to rather fainter luminosities in both the optical/IR and X-ray. However, at late times it does not fall within the locus of X-ray emitting galaxies, either of local galaxies harboring relatively quiescent black holes or of more luminous AGNs. For example, in the comparison of Pineau et al. (2011) of SDSS with 2XMM, only a handful of matches have optical absolute magnitudes fainter than -19 and in most of these galaxies the X-ray luminosity is sufficiently low $\left(10^{38-40} \mathrm{erg} \mathrm{s}^{-1}\right)$ that discrete X-ray emission from binaries, etc. could be responsible for the observed flux. Indeed, the optical absolute magnitude of the host galaxy of Swift J1644+57 of $M_{V} \sim-18.5$ is fainter than the cases of Heinze 2-10 (Reines et al. 2011) or Mrk 709 (Reines et al. 2014), both nearby dwarf galaxies thought to harbor massive black holes. Thus, despite the apparent plateau in $\mathrm{X}$-ray luminosity, this argues against the presence of a standard AGN within the host galaxy, as supported by the absence of obvious AGN features in either optical spectroscopy (see above) or late time radio follow-up (Zauderer et al. 2013). Further X-ray observations over increasingly long time periods should ultimately offer a sensitive test of any variability within the source. 


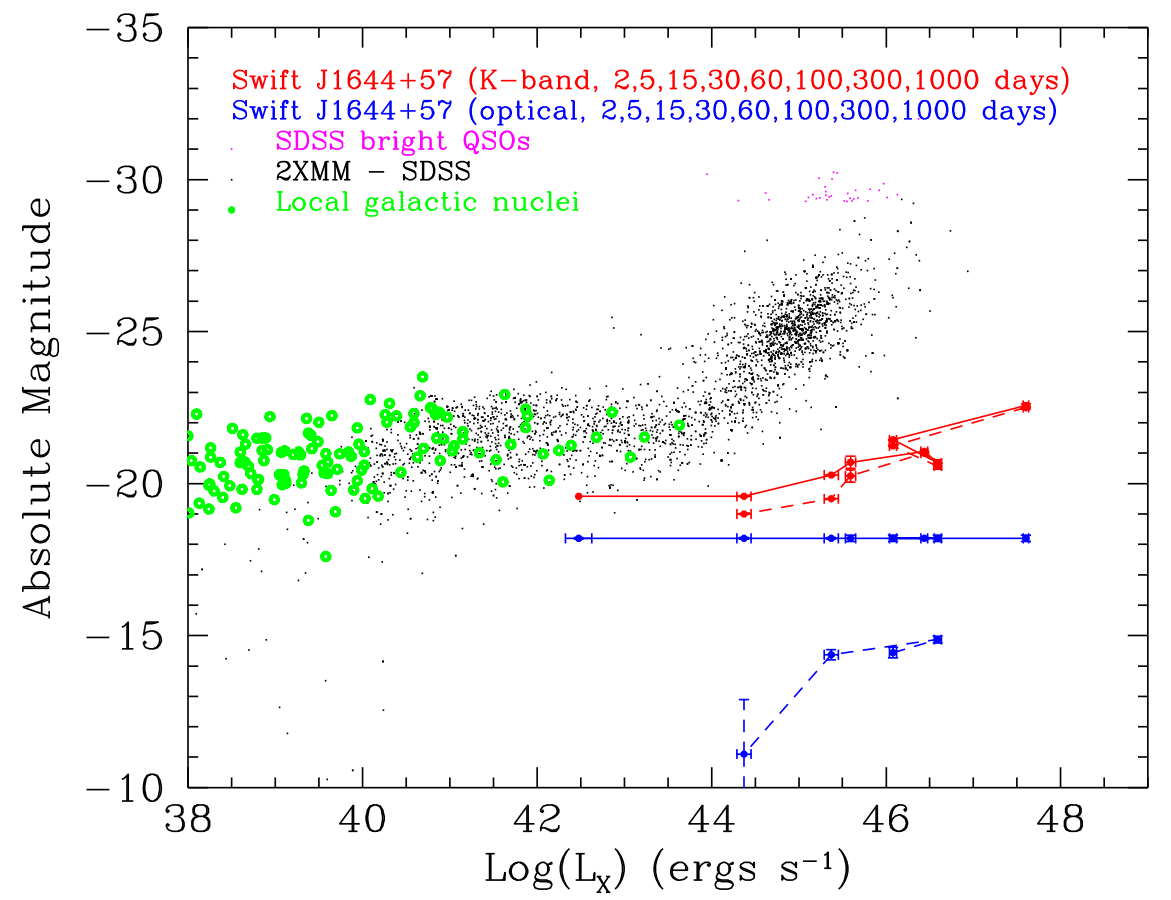

Figure 3. Evolution of the location of the transient in the $L_{\mathrm{X}}-M_{\mathrm{opt} / \mathrm{IR}}$ plane, showing the infrared and optical fading over several years following the first outburst. While at early times the source occupied a region of parameter space largely distinct from that of other transients, its final location is much closer to the locale of normal galaxies. However, it remains unusually X-ray luminous given its optical absolute magnitude. The solid lines represent the total observed light (host galaxy plus transient), while the dashed lines show the host-subtracted transient light not corrected for host galaxy extinction. The final optical counterpart point is plotted as the measured flux with an associated error bar, but could also be represented as an upper limit of $M_{r} \sim-13.5(2 \sigma)$. The X-ray error bars are approximate and account for both the photon noise in X-ray images and the fact that the X-ray source shows some degree of small scale variability; particularly at late times the X-ray and optical observations are not simultaneous.

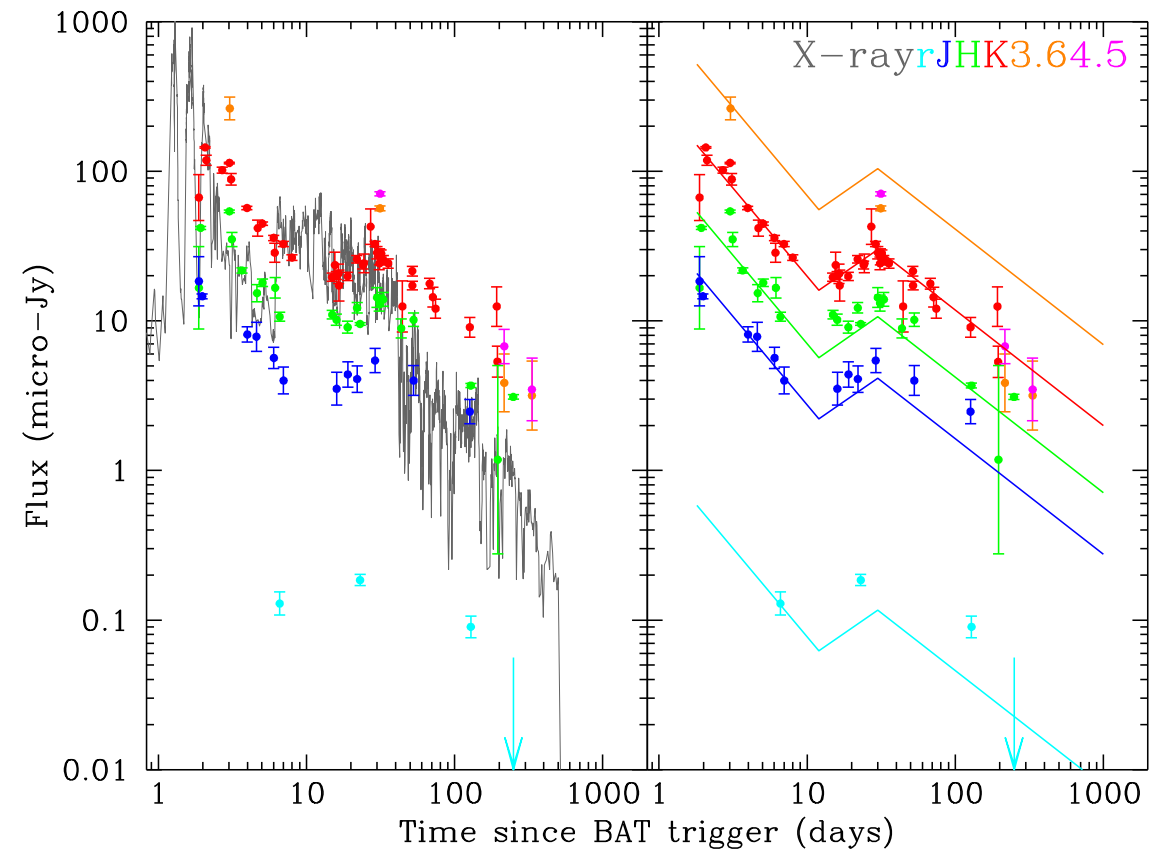

Figure 4. Optical and infrared photometry of Swift $\mathrm{J} 1644+57$ with the contribution of the host galaxy subtracted. The early time behavior is apparently achromatic, with a constant offset between the bands up to 10 days after the BAT trigger, although some variability is visible on top of a gradual decay. After 10 days the counterpart re-brightens to a bump that peaks 30-50 days after trigger. The left-hand panel shows the X-ray light curve for comparison; although there is a significant re-brightening in X-rays, it occurs well before the optical/IR brightening and is much sharper. The optical bump feature is also shown in all the available bands, although it is clearly stronger in the bluer bands. After the peak the behavior is apparently chromatic, with the redder bands falling more rapidly than the blue.

\subsection{Optical/IR Light Curve}

A striking feature of the optical/IR light curves is the presence of an apparent upturn to a peak in the light curve around 30 days after the outburst. Initially the plateauing seen at these times was assumed to be due to the source fading into its host galaxy light, but later observations clearly demonstrate further fading by a factor of $>3$ from this time. There is 
significant point-to-point scatter in the IR observations at many epochs, possibly due to intrinsic variation in the source on short timescales. Direct comparison of observations taken with the same instrument and telescope combination implies that this variability is real, at least at early times. There is also likely to be some scatter due to slight systematic differences between our photometry and that reported by Burrows et al. (2011). This means that as with the X-ray, simple fits to the data do not yield high-quality fits and will provide only an approximation of the true behavior. However, the host-subtracted K-band data can be described by a multiply broken power law as shown in Figure 4. The counterpart declines with $\alpha_{1} \approx 1.3$ (where $F_{\nu} \propto t^{-\alpha}$ ), then rises with $\alpha_{2} \approx-0.7$ to a peak at 30 days. From this point a decline with $\alpha_{3} \approx 0.8$ describes the final fading into the host galaxy, although there are significant errors on the late time points due to the uncertainty in host subtraction. This crude model of three power-law segments also provides a reasonable fit to the $\mathrm{H}$ - and J-band observations if an arbitrary offset is applied (see Figure 4). If this offset is scaled to provide a good match to the early data $(<10$ days post-burst) then it significantly underpredicts the strength of the bump in the $\mathrm{H}$ and $\mathrm{J}$ bands. This suggests that the bump does not have the same underlying spectral energy distribution (SED) as the earlier counterpart and is much bluer with relatively weak IR emission.

The HST observations provide the best measurements of this bump since they can cleanly be subtracted for host contribution without the need for PSF matching or differences between cameras or filters. However, the HST observations also provide extremely poor temporal sampling. Nonetheless, it is striking to note that the F606W optical observations show an apparent rise between 6 and 23, with the 23 day flux $\sim 1.5$ times brighter than at day 6 , while the IR light at 23 days is 0.9 times as bright as at 6 days (see Figure 5). This offers further evidence that the bump is a separate feature rather than a simple, achromatic rebrightening. The HST observations also suggest that at later times the decay cannot be well fit as a single power-law decay, although this is again based on very small sampling (3 points per band).

In Figure 6 we show the evolution of the SED of Swift J1644 +57 . It can be seen to be extremely red, as previously noted. Its SED, combined with the significant X-ray column density, favors an optical extinction in the region $1.5<E(B-V)<2$ (Bloom et al. 2011b; Burrows et al. 2011; Levan et al. 2011). To highlight the possible impact of extinction, we then also plot the SED corrected for a maximal extinction of $E(B-V)=2$ assuming a Milky Way extinction law, although since none of our wavelengths are close to the rest-frame $2175 \AA$ bump, the choice of extinction law has minimal impact on the correction. The peak of the bump at 30 days has an absolute magnitude of $M_{B} \sim-22$ for a maximal extinction, comparable to the peak magnitude in the $\mathrm{K}$ band (which is far less affected by host extinction).

\subsection{The Origin of the Optical Bump}

Perhaps the most plausible explanation is that the optical bump originates as the hot thermal component of the tidal flare. Such components are typically those expected based on nonrelativistic models (e.g., Rees 1988). This peak luminosity occurs well after the disruption itself since the peak accretion rate is after the return of the most bound debris. Indeed, numerical models of mass return suggest that luminous UV flares may peak on timescales of $\sim 20-50$ days at optical and UV wavelengths (Lodato \& Rossi 2011) with luminosities rather similar to those of normal SNe. This is broadly borne out by observations of candidate disruptions to date, with many of the most promising candidates showing such rises. However, suggested examples of TDFs actually show a surprisingly large variation in their properties. Some peak early and very bright $\left(M_{V}<-20\right.$ and rise times of a few days, e.g., PTF10iya (Cenko et al. 2012a)) while those with much longer lifespans are also significantly fainter $\left(M_{V}>-18\right.$ with rise times of 20-50 days). There are no examples that apparently match the energy output for Swift J1644+57, although there remains significant uncertainty about both the extinction and the contribution of any non-thermal component. We plot the light curves of Swift J1644+57 (after subtraction of the host contribution and correction for host extinction) against those of candidate TDFs in Figure 5. Unfortunately, such a comparison is non-trivial since Swift J1644+57 is predominantly observed in the rest-frame IR, while the thermal flares are strong UV and optical emitters. Therefore, the poorly sampled optical light curve of Swift J1644+57 (cyan line in Figure 5) is probably the best comparison with known examples. However, the strong evolution in the optical/IR SED of the source occurs primarily at early times, where the IR light is falling as the optical rises. As this is before the peak of the bump, we can compare the later evolution by scaling the well sampled K-band light curve to the optical. It should also be noted that this comparison is further complicated since the origin of many suggested TDFs remains uncertain, for example, some may be unusual $\mathrm{SNe}$ and others due to partial disruption (e.g., Chornock et al. 2014; Holoien et al. 2014) or the disruption of unusual stars (e.g., Gezari et al. 2012).

Another possibility is that the re-brightening is due to the optical/IR contribution of the second synchrotron component identified by Berger et al. (2012). This peaks at radio wavelengths at $\sim 100$ days, although plausible synchrotron models could result in an earlier peak for the optical/IR emission (as seen in GRBs for example (Sari et al. 1998)), depending on the location of spectral breaks. This would have the appeal of representing the manifestation of a feature known at other wavelengths and might also explain the relatively high polarization $(7.4 \pm 3.5 \%)$ seen in the IR 17 days after the outburst as the bump is beginning to dominate (Wiersema et al. 2012). Indeed, as noted by De Colle et al. (2012), the delay between viewing energy injection at the base of the jet in $\mathrm{X}$-rays and radio emission from the jet head is naturally expected in models of jetted TDFs, and this "lag" in which the optical/IR peak is between these two extremes may have some appeal. However, the parameters would necessarily require some tuning to provide the brightening without the presence of any moving spectral breaks in the optical/IR since while the relative strength of the bump emission varies with wavelength, the shape of the bump is broadly similar. The bump colors would also be unusually blue-corrected for host extinction: the spectrum would follow $F_{\nu} \sim \nu^{2}$ or steeper, much bluer than expected for GRB-blast waves in this wavelength regime. The polarization measurement could also represent underlying asymmetry in the source, as is seen in some $\mathrm{SNe}$ (e.g., Patat et al. 2011), while its intrinsic value is significantly uncertain since interstellar polarization within the host could also play an important role (Wiersema et al. 2012). To date there do not 


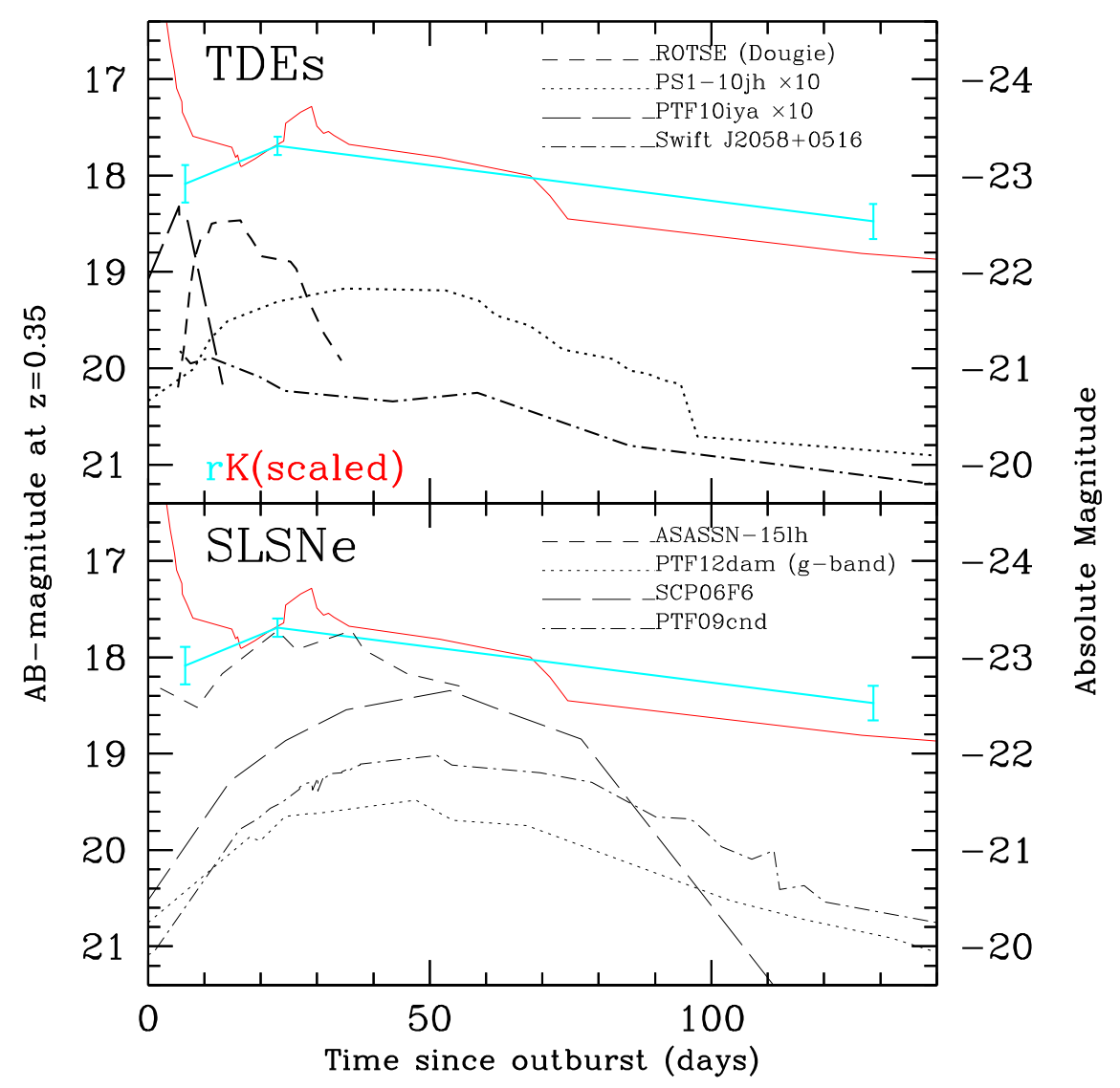

Figure 5. Comparison of the host subtracted, extinction corrected light curves of Swift J1644+57 with other luminous transient events, in particular the light curves of suggested candidate tidal disruption systems (top, including the luminous "Dougie" discovered by ROTSE (Vinkó et al. 2015), PS1-10jh (Gezari et al. 2012), PTF10iya (Cenko et al. 2012a), and Swift J2058+0516 (Cenko et al. 2012b; Pasham et al. 2015)) and SLSNe (bottom, including ASASSN-15lh (Dong et al. 2016), PTF12dam (Nicholl et al. 2013), SCP 06F6 (Barbary et al. 2009), and PTF09cnd (Quimby et al. 2011)). The left axis shows the events as they would appear at the same redshift as Swift J1644+57, and the right-hand axis shows absolute magnitudes. Unfortunately, the poor sampling of the optical component of Swift J1644+57 (cyan line) makes a direct comparison with the predominantly optical observations of other transient classes difficult. To attempt to mitigate this we have also shown the smoothed K-band light curve, scaled to the optical at 23 days. The strong chromatic evolution at early times can be seen with the optical rising as the $\mathrm{K}$ band falls. However, around and after the peak, this suggests that the overall light curve shape suggested by the optical observations is probably a reasonable expectation. However, SLSNe can provide a reasonable match to the observations (in particular ASASSN-15lh), while TDFs match the light curve shape, but are required to be significantly brighter than previous examples.

exist polarimetric observations of the thermal components of TDF flares, and so this cannot be compared directly.

An alternative hypothesis is that the optical bump could be due to reverberation of the X-ray light. Yoon et al. (2015) claim that the morphology of the optical is similar to that of the $\mathrm{X}$-ray, but with a delay of $\sim 15$ days. While this does not appear the case in a detailed comparison (for example, the $\mathrm{X}$-ray rise is rapid while the optical/IR rise apparently takes place over the timescale of several days) it is possible that a prompt injection of energy in the X-ray could be smoothed out should the reverberating material be spread out at an average distance of $\sim 15$ light days from the central engine. While the lags to the broadline region can be of this size (Peterson et al. 2004) simultaneous optical/X-ray monitoring of AGNs typically yields much smaller lags ( $\sim 1$ day) between X-ray and optical emission (e.g., Breedt et al. 2009, 2010), while lags due to processes within the disk are also short $(\sim 1$ day) and should increase with increasing wavelength (McHardy et al. 2014). Hence the properties of the light curves do not naturally match the expectations of reverberation seen in AGNs and would require an unusual, pre-existing AGN-like geometry to exist within the host. On the other hand, the unexpectedly high optical luminosities and low effective temperatures of many optically selected TDFs have also been attributed to "reprocessing" of the inner disk emission by debris from the merger, either bound debris still returning to the $\mathrm{BH}$ (Guillochon et al. 2014) or an unbound outflow from the accretion disk (e.g., Strubbe \& Quataert 2011; Metzger \& Stone 2015).

The other class of astrophysical transient that can reach such extreme luminosities are the superluminous supernovae (SLSNe; e.g., Gal-Yam 2012). These events peak at magnitudes of $M_{V}<-21$ and have slow rise times of 30-100 days, followed by slow decays. The peak luminosity of Swift J1644 +57 is comparable to these events and given the uncertainty in both the explosion date of SLSNe and the true "trigger" time for Swift $\mathrm{J} 1644+57$, it is possible to obtain a reasonable match in both light curve shape and luminosity. For the case of $E$ $(B-V)=2$ the luminosity would be among the highest for SLSNe, although the recent discovery of the most luminous SLSNe ASASSN-15lh would be comparable (in fact, it should also be noted that ASASSN-15h is apparently coincident with the nucleus of its host galaxy (Dong et al. 2016), as is the second brightest SLSNe, CSS 100217, (Drake et al. 2011), perhaps offering further hints of similarities between classes of astrophysical transient). Given the uncertainties in host extinction one can also find a reasonable match in terms of 

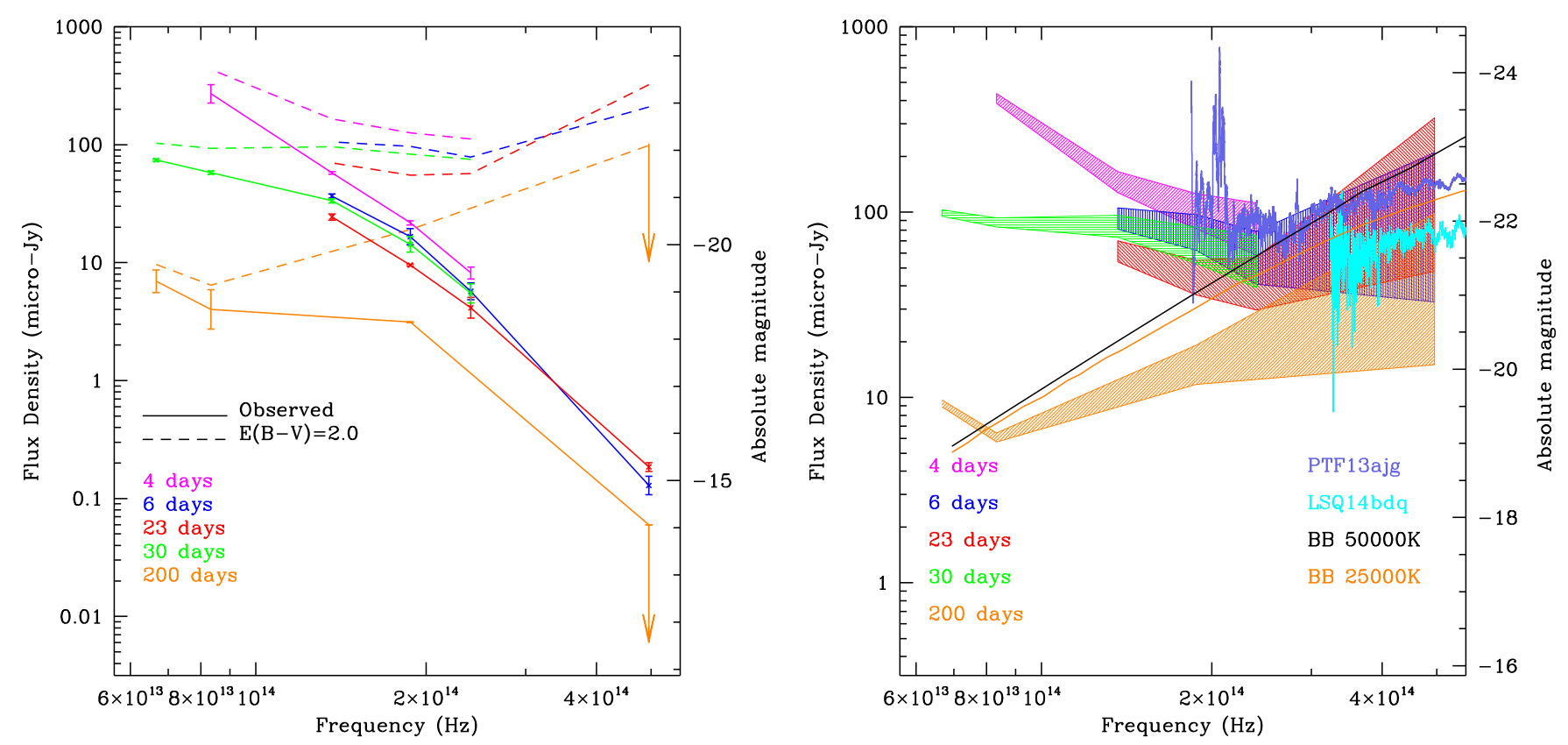

Figure 6. Spectral energy distribution of the Swift J1644+57 at four representative epochs spanning the 200 days after outburst. The left-hand panel shows the multiple epochs as observed (solid lines) and corrected for $E(B-V)_{\text {host }}=2$ (dashed lines). The right-hand panel shows the extinction-corrected SED (the shaded region represents the range between $E(B-V)_{\text {host }}=2$ and $\left.E(B-V)_{\text {host }}=1.5\right)$ in comparison with two representative lines of SLSNe, in particular PTF13ajg (blue) (Vreeswijk et al. 2014) and PTF14bdq (cyan) (Nicholl et al. 2015), taken from WISEREP (Yaron \& Gal-Yam 2012), as well as blackbodies of two different temperatures.

spectral shape between hydrogen poor SLSNe and Swift J1644 +57 (see Figure 6).

At first sight, the strong simultaneous X-ray emission would appear to rule out an SLSNe origin, however, two recent developments may be important in this regard. First, the apparently normal hydrogen poor SLSNe, SCP 06F6 has a strong X-ray detection $>100$ days after its discovery, with a luminosity very similar to that of Swift J1644+57 at the same epoch (Gänsicke et al. 2009; Levan et al. 2013). X-ray observations were not obtained of SCP 06F6 until very late, but it is possible that it is due to jetlike emission that could have been persistent but undetected over a long period in a system similar to Swift $\mathrm{J} 1644+57$. Although it is also possible that the X-ray detection of SCP 06F6 was due to a shorter breakout of magnetar emission (Levan et al. 2013) and the possibilities cannot be distinguished between with the paucity of earlier X-ray observations. Second, in the case of one ultra-long GRB, GRB 111209A (Gendre et al. 2013; Levan et al. 2014) there has recently been the identification of a luminous supernova signature (Greiner et al. 2015), indicating that one can simultaneously observe strong X-ray emission and a luminous $\mathrm{SNe}$ bump. If these $\mathrm{SNe}$ are in fact powered by either a black hole or long-lived magnetar central engines then one might expect to sometimes observe them down a jet axis, in which case events like Swift J1644+57 or GRB 111209A could be observed. Motivated in part by these results Metzger et al. (2015) have shown that the full variety of luminous SNe and extremely long-lived high-energy transients can be explained (although not necessarily uniquely) by magnetars with differing magnetic fields and spin-down times, extending the suggestion by Mazzali et al. (2014) that most GRBs can be explained by such a mechanism. Indeed, they note that this model would naturally predict the luminosity of Swift $\mathbf{J} 2058+0516$. The case of Swift J1644+57 would then also fit on the extrapolation of these models.
Indeed, it is instructive to consider Swift J2058+0516 in this regard, since it exhibited similar high-energy properties to Swift $\mathrm{J} 1644+57$, but lacked the heavy extinction. Thus we might expect to be able to test any SN hypothesis, especially as the redshift was almost identical to the SLSNe SCP 06F6. In this case the luminosity of the optical afterglow was comparable to SLSNe, and the inferred temperature $\left(T \sim 2 \times 10^{4} \mathrm{~K}\right.$, Pasham et al. 2015) was similar to both GRB $111209 \mathrm{~A} / \mathrm{SN} 2011 \mathrm{kl}$ and ASASSN-15lh (Dong et al. 2016; Greiner et al. 2015). However, there was only rather minimal evidence of any optical rise (although observations started late) and optical spectroscopy did not yield any sign of the strong absorption features seen in most SLSNe. This casts some doubt on any model linking events such as Swift J1644+57 and Swift J2058 +0516 with stellar core collapse, although it should equally be noted that in the case of GRB 111209A/SN2011kl (Greiner et al. 2015) the high ejecta velocities diluted any absorption features such that they were not obvious in the observed spectra. The final case of Swift J1112-8238 (Brown et al. 2015) unfortunately does not yield such strong constraints due to rather patchy follow-up, although the absolute magnitude of the transient of $M_{B} \sim-21.4,20$ days after the BAT detection is in keeping with the absolute magnitudes seen in both Swift J1644 +57 and Swift J2058+0516.

Finally, the observed rates of different events could potentially provide some discrimination between progenitor models. Before correction for beaming, GRBs likely show a volumetric rate of a few $\mathrm{Gpc}^{-3}$, corrected for likely beaming this becomes $\sim 300 \mathrm{Gpc}^{-3}$ (e.g., Kanaan \& de Freitas Pacheco 2013), rather comparable to the rate of SLSNe (Quimby et al. 2013). The rate of Swift J1644+57-like transients, or ultra-long GRBs is significantly lower than the GRB rate, although poorly constrained given the small population observed, and observational biases against their detection as long-lived, low peak-flux events (Levan et al. 
Table 4

Host Galaxy Photometry for the Host of Swift J1644+57

\begin{tabular}{lcl}
\hline \hline Band & Mag $(\mathrm{AB})$ & \multicolumn{1}{c}{ References } \\
\hline GALEX/FUV & $>22.1$ & Gezari et al. (2011) \\
GALEX/NUV & $>22.4$ & Gezari et al. (2011) \\
$\mathrm{B}$ & $24.14 \pm 0.05$ & Levan et al. (2011) \\
$\mathrm{g}$ & $23.66 \pm 0.05$ & Levan et al. (2011) \\
$\mathrm{r}$ & $22.80 \pm 0.10$ & Levan et al. (2011) \\
$\mathrm{F} 606 \mathrm{~W}$ & $22.72 \pm 0.03$ & This work \\
$\mathrm{i}$ & $22.31 \pm 0.10$ & Levan et al. (2011) \\
$\mathrm{z}$ & $22.03 \pm 0.03$ & This work \\
$\mathrm{J}$ & $21.87 \pm 0.06$ & This work \\
$\mathrm{H}$ & $21.63 \pm 0.04$ & This work \\
F160W & $21.53 \pm 0.01$ & This work \\
$\mathrm{K}$ & $21.42 \pm 0.04$ & This work \\
Spitzer ch1 & $21.77 \pm 0.27$ & This work \\
Spitzer ch2 & $21.88 \pm 0.24$ & This work \\
WISE W3 & $>17.95$ & Levan et al. (2011) \\
WISE W4 & $>16.14$ & Levan et al. (2011) \\
\hline
\end{tabular}

Note. Since HST observations indicate at early times there was a small transient contribution even in the optical bands we have included an additional error of $0.1 \mathrm{mag}$ on the r- and i-band data.

2014). Brown et al. (2015) estimate a rate of $3 \times 10^{-10}$ galaxy $^{-1} \mathrm{yr}^{-1}$ for Swift J1644+57-like events. Accounting for biases in their detection could give an order of magnitude larger rate, with a similar boost given if shorter events, such as the ultra-long GRBs are included (Levan et al. 2014). Given the volume density of galaxies in the relatively local universe (or more specifically massive black holes) is $\sim 10^{-2}-10^{-3} \mathrm{Mpc}^{-3}$ the inferred volumetric rate of the Swift J1644+57-like events is $\sim 3 \times 10^{-3} \mathrm{Gpc}^{-3} \mathrm{yr}^{-1}$, or allowing for the various selections against their discovery perhaps as high as $\sim 0.1$ $\mathrm{Gpc}^{-3} \mathrm{yr}^{-1}$. Hence, even with very small beaming angles (e.g., the factor of $\sim 10^{2-3}$ needed to bring the observed luminosity below the Eddington limit for a $10^{7-8} M_{\odot}$ black hole) such jets need only be launched from a small fraction of SLSNe. This would explain why evidence for their existence in X-ray monitoring of SLSNe is rare to date (Levan et al. 2013). Equally, these rates are significantly below the rates of TDFs, whose canonical rate of $1 \times 10^{-5}$ galaxy $^{-1} \mathrm{yr}^{-1}$ is 5 orders of magnitude higher than that of the relativistic counterparts. As noted by Cenko et al. (2012b) and Brown et al. (2015) it is therefore unlikely that any significant fraction of TDFs could launch such powerful relativistic jets as seen in Swift J1644+57 and other examples. Overall, the rate arguments suggest that these very long duration transients could arise from some small subset of either TDFs or SLSNe.

\subsection{Host Galaxy Properties}

After the X-ray break it is likely that the observed flux in all bands is now dominated by the host galaxy, affording us the opportunity to investigate it in more detail than previously possible. Indeed, this is supported by the analysis of Yoon et al. (2015) who attempt to fit a point source onto the host, concluding that at later times the point source contribution is minimal. The galaxy is detected in 12 photometric bands from $0.45-4.5 \mu \mathrm{m}$, with upper limits available from GALEX in the
UV (Gezari et al. 2011) and WISE in the mid-IR (Levan et al. 2011), although in practice the upper limits are weak and do not aid in the determination of galaxy properties since they lie well above any plausible galaxy model fitted through the bands in which the host is detected. The photometric detections and limits are shown in Table 4. From this we can derive the physical properties of the host galaxy based on template fitting to the available SED shown in Figure 7. Considering the Binary Population and Spectral Synthesis (BPASS) library of models (Eldridge \& Stanway 2009), we find the SED to be well reproduced by a relatively old dominant stellar population (age $=3.2 \times 10^{9}$ years), although the emission lines clearly indicate the presence of a younger population as well (Figure 7). Importantly, the fitting also provides a much more robust determination of the stellar mass than was previously possible, since earlier attempts were significantly contaminated at red wavelengths by transient light. Specifically we find a stellar mass of $M_{*}=5.5 \times 10^{9} M_{\odot}$. This value is somewhat larger than that found by Yoon et al. (2015) from their more detailed study $\left(M_{*}=1.38_{-0.27}^{+0.48} \times 10^{9}\right)$. However, this may be explained by the use of differing spectral models, and our use of later time Spitzer observations, free from transient contamination. This stellar mass can be used to infer an approximate mass for the central black hole. Scott et al. (2013) find that for core-Sérsic profiles the scaling is roughly linear $\left(M_{\mathrm{BH}} \propto M_{*}^{0.97 \pm 0.14}\right)$, but for galaxies with low masses (they define low to be $M_{*}<3 \times 10^{10} M_{\odot}$ ) they find a much steeper relation of $M_{\mathrm{BH}} \propto M_{*}^{2.22 \pm 0.58}$. Under the assumption that the galaxy stellar mass is equal to its spheroid mass (which seems a reasonable assumption given the surface brightness profile, see below) the implied black hole mass is then $M_{\mathrm{BH}} \sim 3 \times 10^{6} M_{\odot}$, which could be taken as an upper limit on the likely $\mathrm{BH}$ mass.

Despite its luminosity appearing very similar to the LMC $\left(M_{B} \sim-18\right)$, the morphological properties of the host of Swift $\mathrm{J} 1644+57$ are rather different. The core of the galaxy is barely resolved by the HST IR observations, although is reasonably resolved in the optical. The galaxy has little ellipticity $e \approx 0.1$ and is very concentrated, with $R_{20,50,80}=0.077,0.184$, 0 " $388=0.39,0.92,1.95 \mathrm{kpc}$ at $z=0.354$. Its surface brightness profile is well fit with a Sérsic fit with $n=4$ (i.e., a de Vaucouleurs profile) in both the optical and IR, suggesting it is dominated by a spheroidal component (see also Yoon et al. 2015). However, a subtraction of a rotated image does reveal some asymmetry with a knotlike structure extending $\sim 0$ !. 1 from the galaxy nucleus, but interestingly including the location of the transient. These are potentially the star-forming regions creating emission lines and lead to a formal concentrated asymmetry measure of $C \approx 3.5, A \approx 0.1$, placing the host in a region of in the concentration asymmetry plane similar to many GRB hosts (Conselice et al. 2005).

\section{SUMMARY}

We have presented multi-wavelength observations of Swift $\mathrm{J} 1644+57$, continuing for $>4$ years after its initial detection. At this stage the observed light at X-ray, optical, and infrared wavelengths appears to be dominated by quiescent emission. In the case of the optical/IR this is likely the host galaxy. In X-rays, an apparently persistent source of luminosity $L=4 \times 10^{42} \mathrm{erg} \mathrm{s}^{-1}$ either represents a slowly declining phase of the counterpart or an underlying low-luminosity AGN. The presence of an AGN in a tidal disruption event is not 


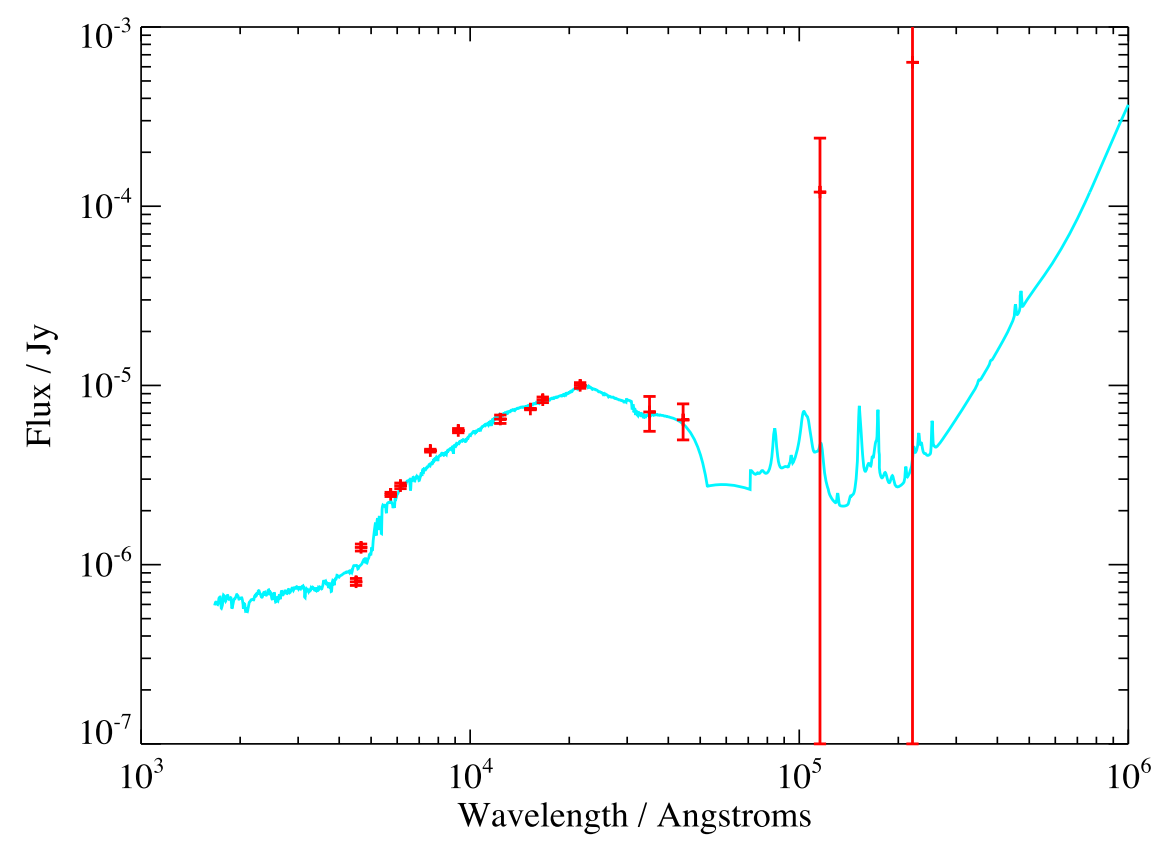

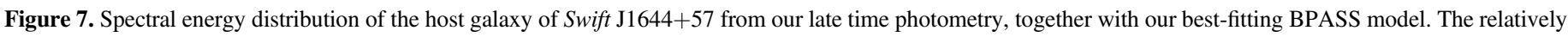

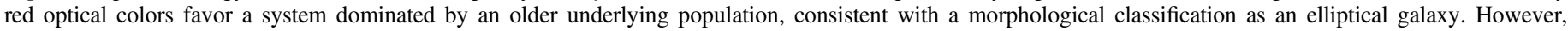
emission lines observed in the optical spectrum demonstrate the presence of some ongoing star formation.

unprecedented; in particular, the recent ASASSN-14li appears to arise from a pre-existing radio galaxy and indeed the coincidence of the source with an apparently active nucleus may increase the confidence in which it can be assigned to a TDF.

More puzzling is the nature of the optical and IR emission. A strong, luminous bump at $\sim 30$ days with an absolute magnitude of $M_{B} \sim-22$ is not well matched by the thermal bumps seen in other tidal flares, since it is much more luminous. The bump is more pronounced than seen in the case of the other candidate flares Swift J2058+0516 and Swift J1112-8238, although this may be due to the earlier initiation of observations in the case of Swift J1644+57. For Swift J1644+57 observations were taken within hours of the BAT trigger, and within at most a few days of the clear onset of activity, in the case of Swift J2058 +0516 and Swift J1112-8238 the first optical observations took place $>10$ and 20 days after the BAT detections respectively, meaning that any rise could have been missed. In all three cases the luminosity of the counterpart is brighter than $M_{B} \sim-21$.

The properties of these bumps may represent extreme versions of the thermal flares from TDFs. In the case of Swift $\mathrm{J} 2058+0516$ the inferred temperature is comparable to those found for thermal TDF flares and the soft X-ray components (Burrows et al. 2011; Levan et al. 2011) may also be consistent with those expectations, although the inferred temperatures of the X-ray blackbodies are much higher than inferred from the optical. Given the apparent differences in total energetics in relativistic TDFs and thermal events it might be less surprising that the thermal bumps are also different and may reflect differences in the stars being accreted (differences in mass, radius, magnetic field, binarity, etc. (e.g., Krolik \& Piran 2011; Mandel \& Levin 2015)). Alternatively, it may be that these events are not in fact from TDFs but from luminous supernovae explosions. In this case they may arise when a luminous SN launches a relativistic jet upon collapse, in which case they would be GRB-like events arising from some subset of SLSNe, as normal long duration GRBs arise from some small subset of SN Ic. The observed rate of SLSNe are comparable to those of GRBs (Quimby et al. 2013), while the rates of the very long transients, even allowing for beaming factors of 100-1000, are much lower, implying that visible high-energy transients associated with SLSNe would be rare, even compared to the SLSNe rate. Metzger \& Stone (2015) develop a model for the optical TDF emission which is qualitatively similar to those developed for engine-powered SLSNe (i.e., reprocessing of central engine energy by approximately a solar mass of outflowing matter; e.g., Dexter \& Kasen 2013), highlighting the challenges of distinguishing TDFs and core collapse events based on their optical light curves alone.

Further diagnostics are clearly needed to form firm conclusions. There are likely to be three routes through which this can come. The first is via spectroscopy of the bumps in any further examples. High quality spectroscopy, allied to detailed modeling can yield diagnostics even in the case of relatively weak or featureless spectra, as recently demonstrated in the case of the ultra-long and luminous supernovae pairing GRB $111209 \mathrm{~A} / \mathrm{SN} 2011 \mathrm{kl}$ (Greiner et al. 2015). The unique identification of features expected in luminous SNe (e.g., turn-off due to line blanketing, absorption lines seen in SLSNe) or TDFs (e.g., blueshifted narrow lines from streams (Strubbe \& Quataert 2011)) would then provide a clinching argument as to the origin of the bumps in the longest high-energy transients. A second route arises through studying the locations of the transients within their hosts. Swift J1644+57 clearly arises very close to the galactic nucleus, and Swift $\mathrm{J} 2058+0516$ is also consistent with the nucleus of a much fainter galaxy (Pasham et al. 2015). In the case of GRBs, approximately $1 / 6$ of examples are consistent with a galaxy nucleus (Fruchter et al. 2006; Svensson et al. 2010); this number may be lower for SLSNe (Lunnan et al. 2015) although the origin of SLSNe in the nuclei of galaxies may be ambiguous (e.g., Dong et al. 2016). Further examples, all in the nuclei of their hosts, 
would rapidly remove any $\mathrm{SNe}$ model from consideration. Finally, we can also consider the host galaxy more globally. TDFs can be observed in quiescent, non-star-forming galaxies while SLSNe are thought to arise from massive star collapse (Gal-Yam 2012) and in principle should occur only in star forming galaxies. A prime model for SLSNe is that they arise from supernovae in which the shock wave is re-energized by the spin-down energy of a recently formed magnetar (Kasen \& Bildsten 2010). While magnetars similar to those suggested to power SLSNe can be formed via accretion induced collapse of two merging white dwarfs (Usov 1992; Levan et al. 2006), and may provide a similar energy input, in the case of a white dwarf merger there would be minimal remnant to re-energize, and hence no luminous SNe. This means that the presence of an extremely long event within an quiescent elliptical galaxy would rule out $\mathrm{SNe}$ models, and strongly favor an origin as a relativistic tidal flare. Since a reasonable fraction $(\sim 50 \%)$ of candidate tidal disruptions arise from passive systems, (i.e., those with little sign of star formation) (e.g., Arcavi et al. 2014) such a test should be possible with only a handful of additional examples since we would expect to observe an example in a system without star formation in the near future.

We thank the referee for constructive comments on the paper. A. J. L., N. R. T., K. W., and P. T. O. thank STFC for support. K. L. P. thanks the UK Space Agency. We thank Matt Mountain, Harvey Tannenbaum, and Norbert Schartel and the teams from STScI, CXC, and ESAC for the approval and rapid scheduling of DDT observations with HST, Chandra and $X M M$-Newton respectively.

Based on observations made with the NASA/ESA Hubble Space Telescope, obtained [from the Data Archive] at the Space Telescope Science Institute, which is operated by the Association of Universities for Research in Astronomy, Inc., under NASA contract NAS 5-26555. These observations are associated with $H S T$ programs GO 12447, 12378 and 12764.

The scientific results reported in this article are based to a significant degree on observations made by the Chandra X-ray Observatory. The observations reported are from program numbers 12900486, 13708437, and 15700509.

Based on observations obtained with XMM-Newton, an ESA science mission with instruments and contributions directly funded by ESA Member States and NASA.

Based on observations obtained at the Gemini Observatory, which is operated by the Association of Universities for Research in Astronomy, Inc., under a cooperative agreement with the NSF on behalf of the Gemini partnership: the National Science Foundation (United States), the National Research Council (Canada), CONICYT (Chile), the Australian Research Council (Australia), Ministério da Ciência, Tecnologia e Inovação (Brazil), and Ministerio de Ciencia, Tecnología e Innovación Productiva (Argentina).

This work made use of data supplied by the UK Swift Science Data Centre at the University of Leicester, funded by the UK Space Agency.

\section{REFERENCES}

Anderson, J. 2014, BAAS, 224, 12205

Arcavi, I., Gal-Yam, A., Sullivan, M., et al. 2014, ApJ, 793, 38

Barbary, K., Dawson, K. S., Tokita, K., et al. 2009, ApJ, 690, 1358

Berger, E., Zauderer, A., Pooley, G. G., et al. 2012, ApJ, 748, 36

Bloom, J. S., Butler, N. R., Cenko, S. B., \& Perley, D. A. 2011a, GCN 11847,1
Bloom, J. S., Giannios, D., Metzger, B. D., et al. 2011b, Sci, 333, 203 Breedt, E., Arévalo, P., McHardy, I. M., et al. 2009, MNRAS, 394, 427 Breedt, E., McHardy, I. M., Arévalo, P., et al. 2010, MNRAS, 403, 605 Brown, G. C., Levan, A. J., Stanway, E. R., et al. 2015, MNRAS, 452, 4297 Burrows, D. N., Kennea, J. A., Ghisellini, G., et al. 2011, Natur, 476, 421 Cannizzo, J. K., Lee, H. M., \& Goodman, J. 1990, ApJ, 351, 38

Cannizzo, J. K., Troja, E., \& Lodato, G. 2011, ApJ, 742, 32

Cenko, S. B., Bloom, J. S., Kulkarni, S. R., et al. 2012a, MNRAS, 420, 2684

Cenko, S. B., Krimm, H. A., Horesh, A., et al. 2012b, ApJ, 753, 77

Chornock, R., Berger, E., Gezari, S., et al. 2014, ApJ, 780, 44

Conselice, C. J., Vreeswijk, P. M., Fruchter, A. S., et al. 2005, ApJ, 633, 29

Cummings, J. R., Barthelmy, S. D., Beardmore, A. P., et al. 2011, GCN, 11823,1

De Colle, F., Guillochon, J., Naiman, J., \& Ramirez-Ruiz, E. 2012, ApJ, 760,103

Dexter, J., \& Kasen, D. 2013, ApJ, 772, 30

Dong, S., Shappee, B. J., Prieto, J. L., et al. 2016, Sci, 351, 257

Drake, A. J., Djorgovski, S. G., Mahabal, A., et al. 2011, ApJ, 735, 106

Eldridge, J. J., \& Stanway, E. R. 2009, MNRAS, 400, 1019

Evans, P. A., Beardmore, A. P., Page, K. L., et al. 2007, A\&A, 469, 379

Evans, P. A., Willingale, R., Osborne, J. P., et al. 2010, A\&A, 519, A102

Fruchter, A. S., \& Hook, R. N. 2002, PASP, 114, 144

Fruchter, A. S., Levan, A. J., Strolger, L., et al. 2006, Natur, 441, 463

Gal-Yam, A. 2012, Sci, 337, 927

Gänsicke, B. T., Levan, A. J., Marsh, T. R., \& Wheatley, P. J. 2009, ApJL, 697, L129

Gendre, B., Stratta, G., Atteia, J. L., et al. 2013, ApJ, 766, 30

Gezari, S., Chornock, R., Rest, A., et al. 2012, Natur, 485, 217

Gezari, S., Forster, K., \& Martin, D. C. 2011, ATel, 3292, 1

Giannios, D., \& Metzger, B. D. 2011, MNRAS, 416, 2102

Greiner, J., Mazzali, P. A., Kann, D. A., et al. 2015, Natur, 523, 189

Guillochon, J., Manukian, H., \& Ramirez-Ruiz, E. 2014, ApJ, 783, 23

Holoien, T. W.-S., Prieto, J. L., Bersier, D., et al. 2014, MNRAS, 445, 3263

Kanaan, C., \& de Freitas Pacheco, J. A. 2013, A\&A, 559, A64

Kasen, D., \& Bildsten, L. 2010, ApJ, 717, 245

Kennea, J. A., Romano, P., Krimm, H. A., et al. 2011, ATel, 3242,

Komossa, S. 2015, JHEAp, 7, 148

Krimm, H. A., \& Barthelmy, S. D. 2011, GCN, 11891, 1

Krolik, J. H., \& Piran, T. 2011, ApJ, 743, 134

Levan, A. J. 2015, JHEAp, 7, 44

Levan, A. J., Read, A. M., Metzger, B. D., Wheatley, P. J., \& Tanvir, N. R. 2013, ApJ, 771, 136

Levan, A. J., \& Tanvir, N. R. 2012, ATel, 4610,

Levan, A. J., Tanvir, N. R., Cenko, S. B., et al. 2011, Sci, 333, 199

Levan, A. J., Tanvir, N. R., Starling, R. L. C., et al. 2014, ApJ, 781, 13

Levan, A. J., Wynn, G. A., Chapman, R., et al. 2006, MNRAS, 368, L1

Lodato, G., \& Rossi, E. M. 2011, MNRAS, 410, 359

Lunnan, R., Chornock, R., Berger, E., et al. 2015, ApJ, 804, 90

MacLeod, M., Guillochon, J., Ramirez-Ruiz, E., Kasen, D., \& Rosswog, S. 2015a, arXiv:1508.02399

MacLeod, M., Trenti, M., \& Ramirez-Ruiz, E. 2015b, arXiv:1508.07000

Mandel, I., \& Levin, Y. 2015, ApJL, 805, L4

Mangano, V., Burrows, D. N., Sbarufatti, B., \& Cannizzo, J. K. 2015, arXiv: 1511.06447

Mazzali, P. A., McFadyen, A. I., Woosley, S. E., Pian, E., \& Tanaka, M. 2014, MNRAS, 443, 67

McHardy, I. M., Cameron, D. T., Dwelly, T., et al. 2014, MNRAS, 444, 1469

Metzger, B. D., Margalit, B., Kasen, D., \& Quataert, E. 2015, MNRAS, 454,3311

Metzger, B. D., \& Stone, N. C. 2015, arXiv:1506.03453

Nakauchi, D., Kashiyama, K., Suwa, Y., \& Nakamura, T. 2013, ApJ, 778, 67

Nicholl, M., Smartt, S. J., Jerkstrand, A., et al. 2013, Natur, 502, 346

Nicholl, M., Smartt, S. J., Jerkstrand, A., et al. 2015, ApJL, 807, L18

Pasham, D. R., Cenko, S. B., Levan, A. J., et al. 2015, ApJ, 805, 68

Patat, F., Taubenberger, S., Benetti, S., Pastorello, A., \& Harutyunyan, A. 2011, A\&A, 527, L6

Peterson, B. M., Ferrarese, L., Gilbert, K. M., et al. 2004, ApJ, 613, 682

Phinney, E. S. 1989, in IAU Symp Proc., Vol. 136, The Center of the Galaxy, ed. M. Morris (Dordrecht: Kluwer), 543

Pineau, F.-X., Motch, C., Carrera, F., et al. 2011, A\&A, 527, A126

Portegies Zwart, S. F., Baumgardt, H., Hut, P., Makino, J., \& McMillan, S. L. W. 2004, Natur, 428, 724

Quataert, E., \& Kasen, D. 2012, MNRAS, 419, L1

Quimby, R. M., Kulkarni, S. R., Kasliwal, M. M., et al. 2011, Natur, 474, 487

Quimby, R. M., Yuan, F., Akerlof, C., \& Wheeler, J. C. 2013, MNRAS, 431,912 
Ramirez-Ruiz, E., \& Rosswog, S. 2009, ApJL, 697, L77

Rees, M. J. 1988, Natur, 333, 523

Reines, A. E. 2014, Natur, 513, 322

Reines, A. E., Plotkin, R. M., Russell, T. D., et al. 2014, ApJL, 787, L30

Reines, A. E., Sivakoff, G. R., Johnson, K. E., \& Brogan, C. L. 2011, Natur, 470, 66

Russell, D. M., Miller-Jones, J. C. A., Maccarone, T. J., et al. 2011, ApJL, 739, L19

Sari, R., Piran, T., \& Narayan, R. 1998, ApJL, 497, L17

Saxton, C. J., Soria, R., Wu, K., \& Kuin, N. P. M. 2012, MNRAS, 422, 1625

Sbarufatti, B., Burrows, D. N., Gehrels, N., \& Kennea, J. A. 2012, ATel, 4398, 1

Scott, N., Graham, A. W., \& Schombert, J. 2013, ApJ, 768, 76

Seth, A. C., van den Bosch, R., Mieske, S., et al. 2014, Natur, 513, 398

Shen, R.-F., \& Matzner, C. D. 2014, ApJ, 784, 87

Stratta, G., Gendre, B., Atteia, J. L., et al. 2013, ApJ, 779, 66

Strubbe, L. E., \& Quataert, E. 2011, MNRAS, 415, 168
Suzuki, D., Tristram, P. J., Kobara, S., et al. 2011, GCN, 11824, 1

Svensson, K. M., Levan, A. J., Tanvir, N. R., Fruchter, A. S., \& Strolger, L.-G. 2010, MNRAS, 405, 57

Tchekhovskoy, A., Metzger, B. D., Giannios, D., \& Kelley, L. Z. 2014, MNRAS, 437, 2744

Usov, V. V. 1992, Natur, 357, 472

van Velzen, S., Körding, E., \& Falcke, H. 2011, MNRAS, 417, L51

Vinkó, J., Yuan, F., Quimby, R. M., et al. 2015, ApJ, 798, 12

Vreeswijk, P. M., Savaglio, S., Gal-Yam, A., et al. 2014, ApJ, 797, 24

Wiersema, K., van der Horst, A. J., Levan, A. J., et al. 2012, MNRAS, 421, 1942

Willingale, R., Starling, R. L. C., Beardmore, A. P., Tanvir, N. R., \& O'Brien, P. T. 2013, MNRAS, 431, 394

Woosley, S. E., \& Heger, A. 2012, ApJ, 752, 32

Yaron, O., \& Gal-Yam, A. 2012, PASP, 124, 668

Yoon, Y., Im, M., Jeon, Y., et al. 2015, ApJ, 808, 96

Zauderer, B. A., Berger, E., Margutti, R., et al. 2013, ApJ, 767, 152

Zauderer, B. A., Berger, E., Soderberg, A. M., et al. 2011, Natur, 476, 425 